\title{
ELM pacing investigations at JET with the new pellet launcher
}

P. T. Lang 1), A. Alonso 2), B. Alper 3), E. Belonohy 1), A. Boboc 3), S.Devaux 1), T.Eich 1), D. Frigione 4), K. Gál 5), L. Garzotti 3), A. Geraud 6), G. Kocsis 5), F. Köchl 3,7), K. Lackner 1), A. Loarte 8), P.J. Lomas 3), M. Maraschek 1), H.W. Müller 1), R. Neu 1), J. Neuhauser 1), G. Petravich 5), G. Saibene 9), J. Schweinzer 1), H.Thomsen 1), M. Tsalas 10), R. Wenninger 11), H. Zohm 1) and JET EFDA Contributors*

JET-EFDA, Culham Science Centre, Abingdon, OX14 3DB, UK

1) MPI für Plasmaphysik, EURATOM Association., 85748 Garching, Germany

2) Laboratorio Nacional de Fusion, Euratom-CIEMAT, 28040 Madrid, Spain

3) Euratom/CCFE Fusion Association, Culham Science Centre, Abingdon, OX14 3DB, UK

4) Associazione EURATOM-ENEA sulla Fusione, CP 65, Frascati, Rome, Italy

5) KFKI RMKI, EURATOM Association, P.O.Box 49, H-1525 Budapest-114, Hungary

6) CEA Cadarache, Association Euratom/CEA, 13108, St Paul-Lez-Durance, France

7) Association EURATOM-ÖAW, Atominstitut, TU Wien, 1020 Wien, Austria

8) ITER Organization, 13067, St Paul-Lez-Durance, France

9) FUSION FOR ENERGY, Joint Undertaking, 08019 Barcelona, Spain

10) JET-EFDA, Culham Science Centre, Abingdon, OX14 3DB, UK

11) Universitätssternwarte der LMU, Scheinerstr. 1, D-81679 München, Germany

E-mail contact of main author: peter.lang@aug.ipp.mpg.de

Short tile: ELM pacing investigations at JET

PACS numbers: 52.35.Py, 52.55.Fa, 52.55.Tn

\begin{abstract}
A new pellet injection system was installed at JET designed for both fuelling and ELM pacing. The purpose of the pacing section was to validate pellet ELM pacing as a suitable tool for ELM mitigation in ITER. Pellet pacing was confirmed at the large size scale of JET. The dynamics of triggered ELMs was investigated with respect to their spontaneous counterparts. Triggered ELMs show features also typical for spontaneous ELMs in several operational regimes. Since none of these regimes was unsettled by the pellets this is a strong hint for compatibility with other plasma control tools. Observations and modelling results indicate the ELM triggering occurs by the pellet ablation plasmoid evolving into the first ELM filament followed by a poloidal spread of the instability. An ELM obviously can be forced by a pellet due to the strong local perturbation imposed already under unusual onset conditions but then evolves like any ELM typical for the according plasma regime. For tool optimization the pellet mass and hence the convective confinement losses imposed have to be minimized. In our experiments, a lower mass threshold was observed for the first time. It has been found that to reliably trigger an ELM the pellet needs to be sufficiently large (and fast) to penetrate close to the pedestal top. Recent investigations are clear steps forward to validate the pellet pacing approach for ITER.
\end{abstract}

\footnotetext{
${ }^{*}$ See the Appendix of F. Romanelli et al., 23rd IAEA Fusion Energy Conference, Daejeon (2010), OV/1-3
} 


\section{Introduction}

Heat loads accompanying type-I Edge Localized Modes (ELMs) can cause a threat on the tokamak divertor target plate. Their destructive potential increases with increasing tokamak size [1], reaching worrying dimensions at ITER. Under type-I ELM-like energy loads significant energy and particle flux reaches the divertor target plate which can be eroded due to vaporization and brittle destruction or loss of the melt layer for metals. Calculations for ELMs with short pulse-width show that ELMs with energy densities larger than $1 \mathrm{MJm}^{-2}$ would lead to an intolerably short divertor target lifetime [2]. Hence a major concern about the proposed baseline type-I ELMy H-Mode scenario for ITER is the magnitude of the energy release from the pedestal region towards the divertor and first wall during type-I ELMs and the resultant surface effects [3]. A present database comparing ELM sizes across devices and plasma configurations [4] would project an ITER ELM size that is far above the tolerable limit for the ITER divertor. Several approaches to solve this problem are considered, one of them is the pacing concept. Hereby, the ELM energy $W_{\text {ELM }}$ is reduced by raising in a controlled manner the ELM frequency $f_{\text {ELM }}$ relying on the empirically observed relation $\mathrm{W}_{\text {ELM }}$ $\times \mathrm{f}_{\mathrm{ELM}}=$ const [1]. Decreasing the ELM energy content, the heat load on the divertor is decreased as well. Successful ELM pacing and mitigation by the injection of cryogenic Deuterium (D) pellets was demonstrated first at ASDEX Upgrade [5] and applied over a broad parameter range. Beside effective heat load reduction to the divertor, scenarios with controlled ELMs are also characterized by impurity expulsion (during intrinsically ELM-free phases), a basic ITER requirement [6]. Although pellet pacing is demonstrated and applied on other tokamaks as well, for example on DIII-D [7], there are still issues with respect to the relevance for ITER. Instead of an ELM frequency enhancement factor (paced $\mathrm{f}^{\mathrm{ELM}} /$ intrinsic $^{\mathrm{E}}$ spontaneous $\mathrm{f}_{\text {ELM }}^{0}$ ) of about $2-5$ achieved yet, ITER request a value in the range $10-30$. In addition, confinement deterioration caused by the unnecessary large convective losses lowered the significance of the earlier experiments as they were hampered by using pellet systems originally designed for fuelling purposes.

To prove ITER relevance of this technique several questions still need to be answered. The most important are: can a suitable frequency enhancement be achieved under ITER relevant conditions? Is the ELM related energy flux to the divertor tiles also reduced? What is the minimum unavoidable inherent fuelling constraint? At the best, pellet pacing should be achieved with large frequency enhancement and also with the smallest possible impact on all the other plasma parameters. Hence, pellet ELM pacing has to use the smallest possible pellets and a shallow penetration.

The smallest possible size for producing, accelerating and transferring cryogenic D pellets reliable to the plasma seems to be set by technical boundary conditions to a volume of about 1 $\mathrm{mm}^{3}$. The relative (fuelling) impact per pellet reduces with machine size due to a more favorable relation of pellet and plasma particle inventory. Furthermore, spontaneous ELM frequencies go down with machine size as well. Hence, pellet ELM pacing at a rate significantly beyond $\mathrm{f}^{0}$ ELM avoiding strong fuelling is expected to be carried out much easier from the point of view of pellet injector technical requirements at a large size tokamak. Accordingly, a new pellet launcher system was installed at JET [8] to implement pacing investigations.

This paper is providing an overview of pellet pacing and ELM trigger investigations during JET experimental campaigns C20 - C27 (2008/2009) as follows. First we will report on the experimental boundary conditions determined by the injector set up and its performance, then we describe the target plasma scenarios and the diagnostic equipment. We continue by presenting results achieved for pacing attempts under ITER relevant conditions. Furthermore dedicated investigations on the trigger physics in a wide range of different plasma scenarios and experiments to sound out the trigger threshold are detailed. A discussion of the status of 
pellet pacing investigations with respect to the ITER validation of this technique concludes the paper.

\section{HFPI and experimental boundary conditions}

\subsection{Performance of the HFPI}

The new High Frequency Pellet Injector (HFPI) has been installed as part of the JET program in support of ITER (JET EP2). The prime task of its mission was defined to demonstrate pellet pacing under ITER like conditions (tenfold frequency enhancement in an ITER like plasma scenario) and to sound out the lower limit of the required pellet size. However, in the first years the design parameters were not met due to a leaking barrel in the injector and some faulty connection parts in the transfer system. Thus, the major goal in the last experimental campaigns became the verification of pellet pacing at JET. Climbing one stage on the step ladder AUG-JET-ITER by confirming the pacing approach at the scale of JET was envisaged to assure ELM triggering by pellets does not depend on the rather strong overall impact in a plasma with moderate total particle and energy contend only but can also work under conditions with about the same local but less total impact like in ITER. Furthermore we aimed to investigate trigger features, in particular try to find conditions under which a pellet can trigger an ELM (and when not) and compare the dynamics of triggered ELMs to their spontaneous counterparts. Essentially, the approach intended to gain a better understanding of the ELM trigger mechanism enabling better settled predictions for ITER.

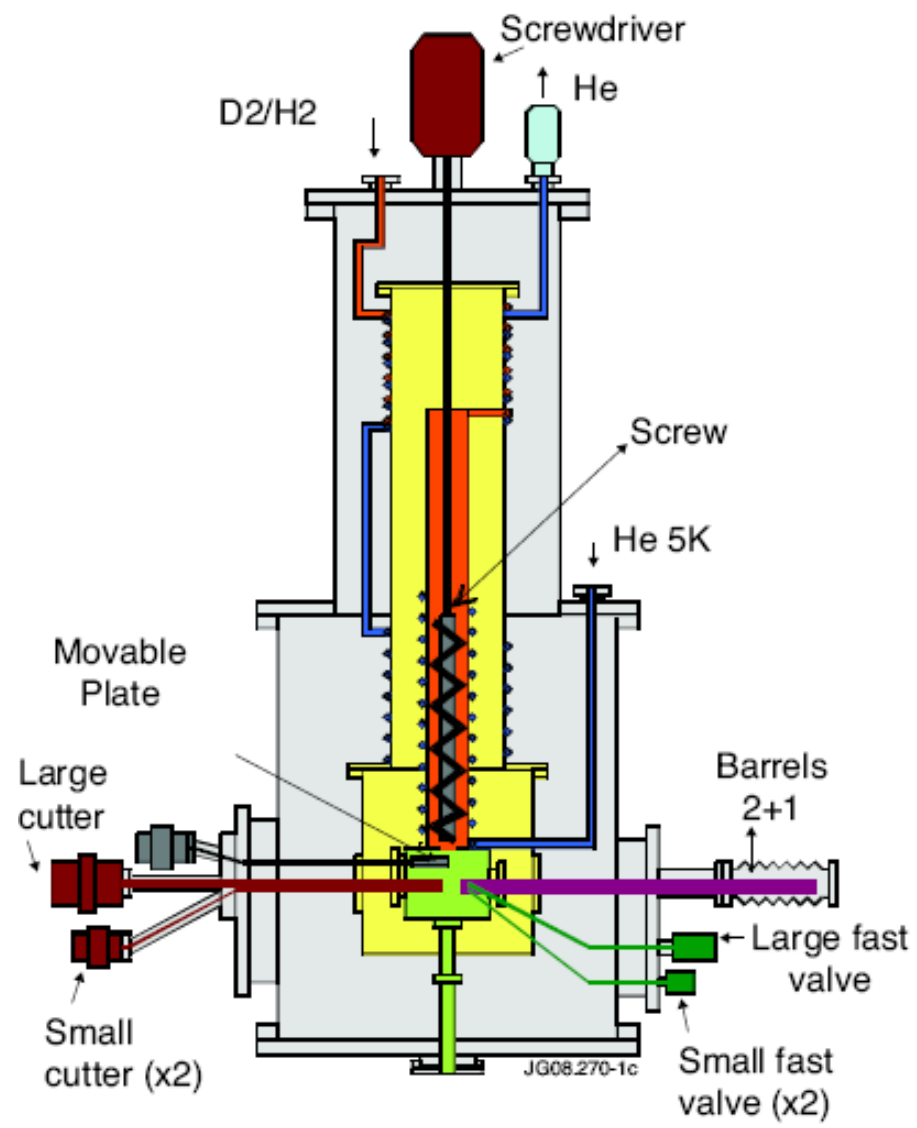

FIG. 1: Schematic drawing of the HFPI. The launcher system is equipped with a screw extruder capable for continuous ice extrusion, delivering either a single rod for production of fuelling size pellets or two rods for pacing pellets. The acceleration section comprises the pellet cutter, a fast gas valve and the according barrel, again a single set for fuelling and a twin set operated in turn for pacing. Due to a leaking barrel the pacing system could be operated only using one barrel. 
The HFPI was designed and built on the basis of the screw extruder technology and pneumatic acceleration using the blower gun concept. It forms an upgrade of a steady state launcher type developed for Tore Supra [9]. The launcher shown schematically in figure 1 was designed to meet both requirements for fuelling and ELM pacing. It comprises two sections operated alternately (no concurrent pacing/fuelling). One is serving on fuelling by delivering pellets from a single extrusion rod with a diameter of $4 \mathrm{~mm}$ to a single acceleration barrel. The pacing section consists of a twin rod extrusion both $1.2 \mathrm{~mm}$ in diameter and their allocated barrels alternately firing pellets in order to double the maximum pellet rate. Nominal pellet size (adjusted by the pellet length) and speed (adjusted by the propellant Helium gas pressure) are fixed for any train injected during an entire plasma discharge. Design parameters are $\mathrm{N}_{\mathrm{P}}=21-42 \times 10^{20} \mathrm{D}$ at $100-500 \mathrm{~m} / \mathrm{s}$ for the fuelling and $0.6-1.2 \times 10^{20} \mathrm{D}$ at $50-200$ $\mathrm{m} / \mathrm{s}$ for the pacing section. Maximum projected repetition rates were 15 and $60 \mathrm{~Hz}$, respectively [8].

The HFPI is connected to the torus by the already existing guiding tube system of the mothballed centrifuge launcher [10] accessing three launching sites inside the torus at Octant 2: outboard or (magnetic) low field side (LFS), inboard or high field side (HFS) and vertical high field side (VHFS). A mechanical selector directs the pellets to one of the tracks or into a dump. Track change during plasma operation is possible within about $0.5 \mathrm{~s}$ allowing usage of different launch positions within a single plasma discharge. In order to cope with the enhanced load on the track system due to a higher level of pellet evaporation and the impact of the propellant gas coming with the new boosted launcher the entire track and pumping system was upgraded accordingly. For example, a purging system flushing with nitrogen had to be incorporated for safety requirements into the most burdened VHFS track. Since the former LFS launch site is covered by the new ITER like ICRH antenna (ILA), the final part of the LFS track inside the torus has been replaced by a new, more bent tube now releasing the pellets in the centre of the antenna. Three diagnostic sections equipped with microwave cavities are incorporated to the guiding system for enabling a monitoring of pellet mass and speed just after pellet launch $(\mu \mathrm{I})$ and just before entering the torus from the LFS $(\mu \mathrm{L})$ or the $\operatorname{VHFS}(\mu \mathrm{V})$.

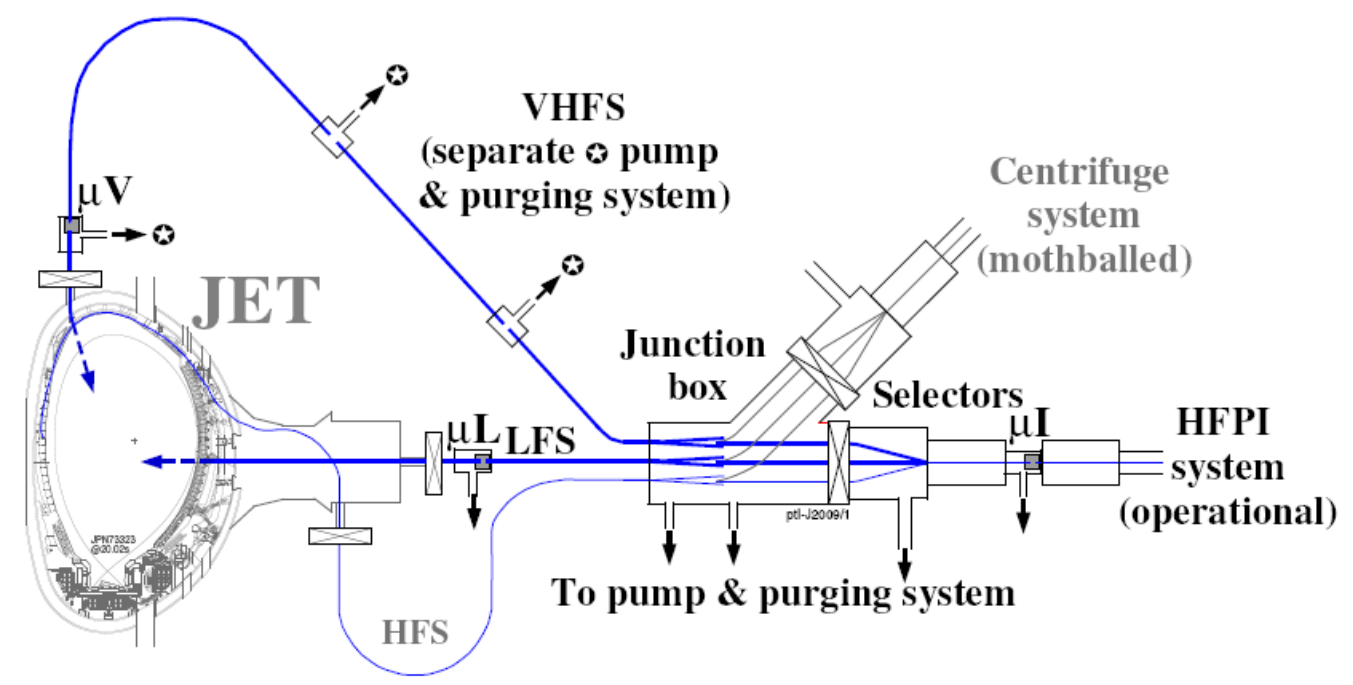

FIG. 2: Launcher set up operated during campaigns C20-C27 (2008/09). Access to the HFS launch track was inhibited for safety reasons, the "Junction box" was simplified by disconnecting the centrifuge before C27. Locations of the three microwave cavities $(\mu I, \mu L$, $\mu V)$ used for pellet mass and velocity measurements are indicated. 
A sketch of the system as put into operation early in 2008 - just before campaign C20 - is shown in figure 2 . In this configuration the entire launching and transfer system did mimic the ITER layout where in a matrix several launchers are connected via tracks, selectors and junction modules to different launching positions inside the torus. To protect the torus against a potential risk of uncontrolled ingress of air pressure monitoring in the guiding tube system close to torus entrance valve is compulsory. Since no monitor was at hand close to HFS torus entrance valve, HFS access had been inhibited for safety reasons. To improve the performance the HFPI system was modified (between campaigns C26 and C27 in April 2009). For example the junction box was simplified by disconnecting the centrifuge in order to enhance the injector capabilities by straightening the tracks and removing some funneling sections. Although major time and resource consuming changes were made in the guiding system the pellet repetition rates and delivery reliability did not achieve the requested values. Hence, the system was used in its "preliminary" form with operational restrictions. Remaining issues are addressed and the system is mended during the 2009-2011 shut down in order to reach its original goals. It is expected to become fully operational for the JET plasma restart with the ITER like wall (ILW, Beryllium wall tiles and Tungsten divertor) [11].
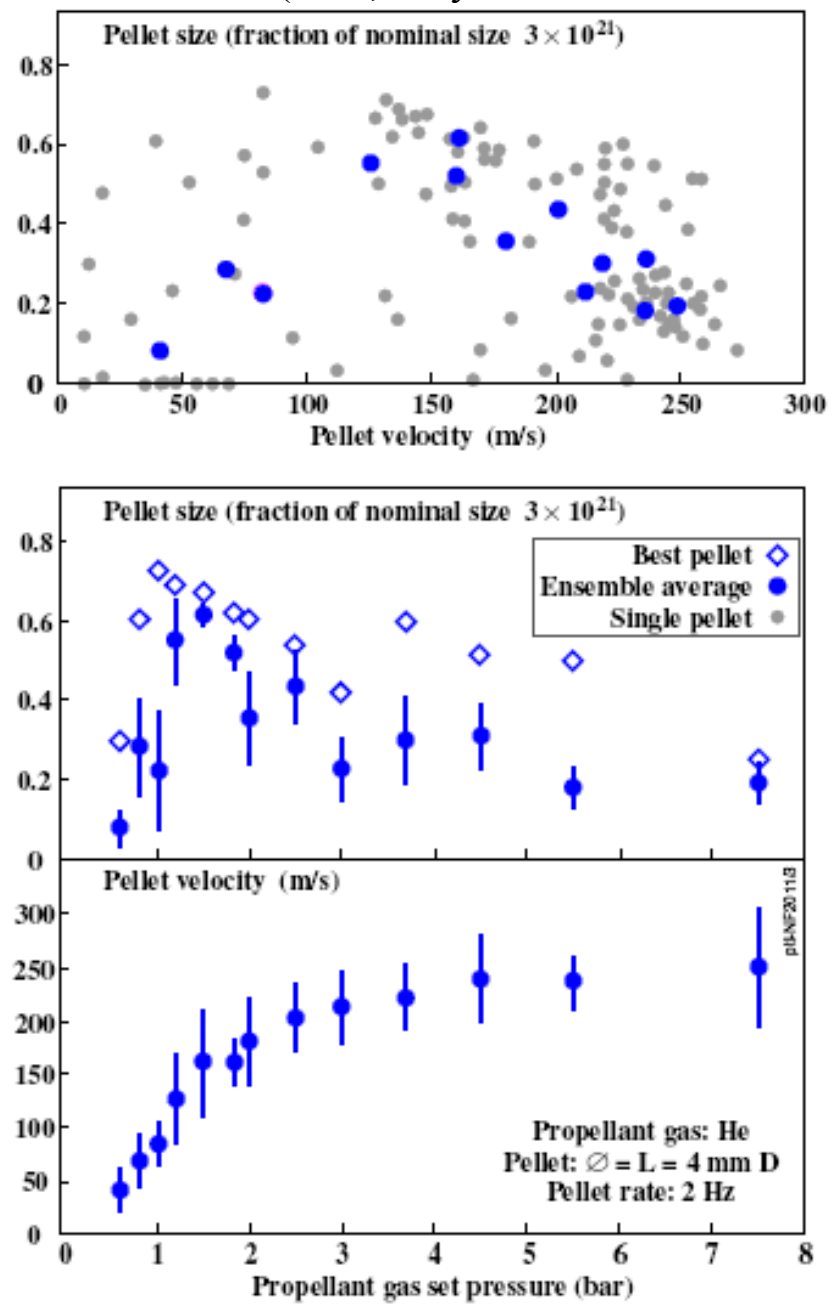

FIG. 3: Launcher system performance achieved for "medium" size fuelling pellets at low repetition rates. Increasing propellant gas pressure rises the pellet speed as expected for the blower gun type acceleration, but stagnation sets in above 3 bars (lower box). Stagnation is connected to higher losses of the pellet mass (data derived from the pellet induced plasma particle inventory enhancement, middle box). The available pellet speed range spans up to almost $300 \mathrm{~m} / \mathrm{s}$, however outside the optimum speed range around $150 \mathrm{~m} / \mathrm{s}$ only at the expense of larger mass scatter. 
For the first campaigns (C20-C26) only the fuelling system was available. The pellet injector was operated with reasonable performance and reliability, but only within a narrow parameter range for LFS launch. Outside this LFS operational window, reliability and performance (size and integrity) decreased. For pellet launch via the VHFS track most $(>70 \%)$ pellets were destroyed during the passage ending up with an emergency locking of the torus valve. Hence, only spurious pellet VHFS launch was possible allowing sample ELM trigger investigations. Sustaining reliable pellet trains could be established solely for LFS launch using pellets of $3 \times$ $10^{21} \mathrm{D}$ nominal particle content in the speed range of $100-200 \mathrm{~m} / \mathrm{s}$ and repetition rates up to $10 \mathrm{~Hz}$. As an example, the evolution of pellet speed and particle content arriving in the plasma for different propellant gas pressures is plotted in figure 3. In this scan, pellets of optimum size were launched from the LFS at a rate of $2 \mathrm{~Hz}$, restricted by the receptiveness of the target plasma; however very similar performance was found for cases using $10 \mathrm{~Hz}$ pellet rate. For pacing experiments relying on the fuelling system, thus $10 \mathrm{~Hz}$ LFS injection was applied with pellet speeds of about $150-200 \mathrm{~m} / \mathrm{s}$, imposing a (real) particle flux of about 1.5 $\times 10^{22} \mathrm{D} / \mathrm{s}$, achieving pellet trains lasting for several seconds with delivery efficiencies (number of pellets arriving in plasma/pellet requests) larger than 0.9-0.95.

For the $\mathrm{C} 27$ campaign the pacing section became also available with a limited repetition rate of $20 \mathrm{~Hz}$ (frequency limitations had to be set due to barrel leaking and restrictions in the ice extrusion speed). The launcher delivered reliable and persisting pellet trains, but many pellets disintegrated during the flight limiting the pellet parameter region. Strong variations were found for the delivery efficiency, best phases reaching up to about 0.4 for LFS and about 0.15 for VHFS launch. Such phases are shown in figure 4. Long lasting trains could be used since the small particle inventory per pellet and moderate rate avoided the excessive pressure rise within the guiding system. Hence pacing and ELM trigger investigations experiments were typically run by applying long phases with pellet launch using the most favorable injector setting. In the case of LFS pellets, occasionally appearing sequences of a few pellets within a $20 \mathrm{~Hz}$ sequence could be obtained. For the VHFS, just occasional pellet impact on the ELM evolution was obtained since $1 / \Delta t_{\text {Pel }}<<f_{\text {ELM }}^{0}\left(\Delta t_{\text {Pel }}\right.$ : temporal distance pellet to pellet, $\mathrm{f}_{\text {ELM }}^{0}$ unperturbed ELM frequency).

\subsection{Experimental boundary conditions}

For the physics investigations JET's versatile diagnostics as well as data processing and validation systems provided a wide range of data. Available diagnostics are very useful for the detection of large pellets in any type of plasmas and for pacing pellets in Ohmic and Lmode plasmas. As shown in figure 4 pellet arrival in a quiescent steady plasma can be monitored from the pellet impact on the plasma edge density as measured by an edge channel of the DCN laser interferometer. However, pacing pellets in type-I ELM phases are hard to detect since ELMs impose a transient behavior with rapidly changing parameters dominating the pellet effect. It should to be noted in this regime of small pellets arriving in a plasma edge undergoing fast strong changes of the local plasma parameters between and even more during an ELM the pellet ablation and penetration can be strongly affected by e.g. variations of the energy flux into the ablation region or by drag effects changing the pellet speed. In order to monitor pellet arrival during H-mode phases dedicated measurements were performed using the (peak) radiation emitted by the pellet cloud. A photo diode allowed recording the total radiation from a region enclosing the VHFS track with high temporal but without spatial resolution. For the LFS launch site, a fast framing camera system was employed [12]. Frame sequences of pre selectable size, spatial and temporal resolution and duration were recorded, the overall duration and resolution limited by the total storage memory available per plasma discharge. An integrated signal of the total ablation radiation like for the VHFS was 
occasionally derived from the frames recorded, however with lower temporal resolution especially for longer trains due to the memory restrictions.

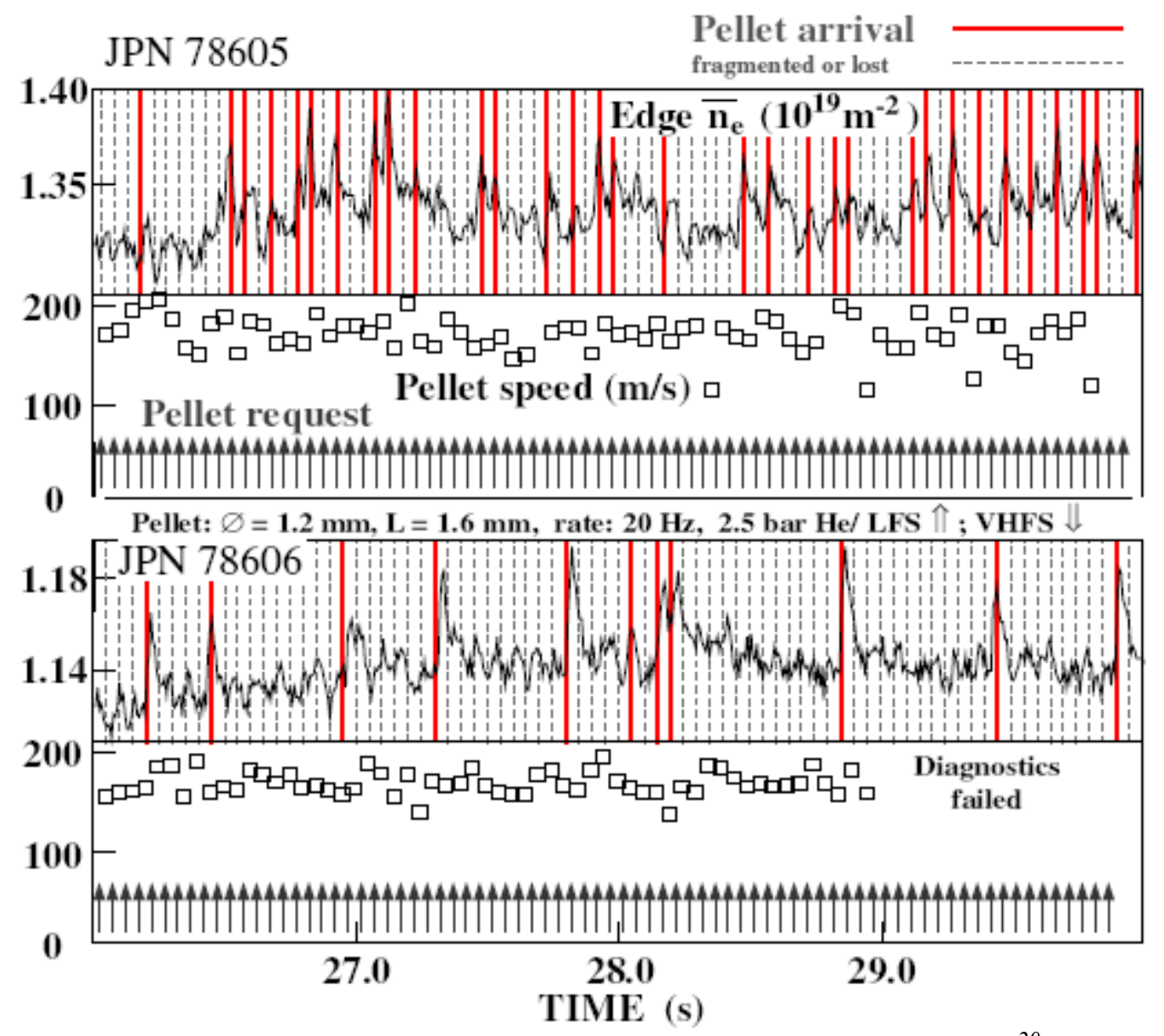

FIG.4 : Monitoring arrival of pacing size pellets (nominal mass $1.1 \times 10^{20} \mathrm{D}$ ) in a quiescent L-mode plasma phase for LFS (upper part) and VHFS site launch. For these $20 \mathrm{~Hz}$ sequences delivery efficiencies of 0.4 and 0.14 are found, respectively.

For the dedicated pacing and triggering experiments we made use of the wide range of plasma configurations developed for the investigation of the ITER baseline scenario in standard ELMy H-mode discharges. From different experiments performed in this context a pool of configurations are at hand known operationally reliable and robust from the point of view of auxiliary heating power, fuelling particle flux, plasma current and shaping. Hence, it was possible to set up desired target discharges with stable low frequency type-I ELMs sufficiently robust for the pellet imposed perturbations. An overview of the experimental configurations used in the study with respect to pellet size, injection locations and plasma configurations is given in Table I. In order to enhance the relative strength of the pellet imposed perturbation and to increase the pellet penetration depth, the plasma (edge) pressure was adjusted by reducing plasma confinement using less shaped (lower triangularity $\delta$ ) and/or lower plasma current. Apart from principal pellet investigations performed in the dedicatedly set up plasma configurations the pellet impact on ELMs was investigated also in a wider range of scenarios. Here, pellet phases were incorporated either as parasitic experiment or for the main purpose of particle fuelling. 


\begin{tabular}{|c|c|c|}
\hline & $\begin{array}{l}\text { Fuelling size } \\
\left(\text { nominal } 30 \times 10^{20} \mathrm{D}\right)\end{array}$ & $\begin{array}{l}\text { Pacing size } \\
\left(\text { nominal } 1.1 \times 10^{20} \mathrm{D}\right)\end{array}$ \\
\hline $\begin{array}{l}\text { LFS (Fast framing camera) } \\
\text { High } \delta(2.5 \mathrm{MA}) \text { "ITER-like" }\end{array}$ & $\begin{array}{l}\text { Up to } 10 \mathrm{~Hz}, \text { reliability } \approx 0.9 \\
\text { JPN76697: } 4 \mathrm{x} \text { ELM frequency } \\
\text { increase with fuelling side effect } \\
\text { (Figure 5) }\end{array}$ & $\begin{array}{l}\text { Up to } 20 \mathrm{~Hz} \text {, reliability } \approx 0.35 \\
\text { JPN78600: Some pellets below } \\
\text { trigger mass threshold } \\
\text { (Figure 13) } \\
\text { JPN78605: Benchmarking of } \\
\text { penetration at trigger mass } \\
\text { threshold } \\
\text { (Figure 15) }\end{array}$ \\
\hline Low $\delta(2.0 \mathrm{MA})$ & $\begin{array}{l}\text { JPN76702: ELM “synchronization" } \\
\text { proofing ELM pacing at JET } \\
\text { (Figure 6) }\end{array}$ & \\
\hline $\begin{array}{l}\text { VHFS (Ablation monitor) } \\
\text { High } \delta(2.5 \mathrm{MA}) \text { "ITER-like" }\end{array}$ & & $\begin{array}{l}\text { Up to } 20 \mathrm{~Hz} \text {, reliability } \approx 0.1 \\
\text { JPN78603: Dynamics of pellet } \\
\text { plasmoid compared to ELMs } \\
\text { (Figure 12) } \\
\text { JPN78606: Determination of } \\
\text { trigger mass threshold } \\
\text { (Figure 14) }\end{array}$ \\
\hline
\end{tabular}

Table I: Overview of typical experimental configurations used during this study with respect to pellet size, injection locations and plasma configurations. Available diagnostics for pellet detection as well as the performance typically achieved is indicated by the shaded text.

Related experiments described later are assigned with reference to figure and JET Pulse Number (JPN).

\section{ELM pacing attempts}

The first pacing attempt using LFS pellet launch was made taking the ITER baseline scenario at a moderate plasma current. This configuration with strong shaping (high triangularity) was developed to achieve good energy confinement at high density and applied in different variants for different investigations. Here a variant was chosen with a low $\mathrm{f}_{\text {ELM }}^{0}$. Pellets of the smallest reliably useful size from the fuelling system were chosen $(3 \mathrm{~mm}$ length, nominal 2.2 $\times 10^{21} \mathrm{D}$ per pellet) at a rate of $10 \mathrm{~Hz}$ and at about $150 \mathrm{~m} / \mathrm{s}$ speed. This came with a slight reduction of delivery efficiency with respect to the peak performance requiring several repetitive attempts in order to achieve a reasonable persistent train as in the example shown in figure 5. The target plasma was run at $\mathrm{I}_{\mathrm{P}}=2.5 \mathrm{MA}$ and $\mathrm{B}_{\mathrm{t}}=2.7 \mathrm{~T}$, yielding $\mathrm{q}_{95}=3.5$. The strong shaping produced an upper triangularity of $\delta_{\mathrm{u}}=0.42$, a lower triangularity of $\delta_{1}=0.40$, the elongation was $\varepsilon=1.76$ and the plasma volume $V_{p}=75 \mathrm{~m}^{3}$. In the initial phase a Neutral Beam was injected at a power of $\mathrm{P}_{\mathrm{NI}}=13 \mathrm{MW}$. Additionally, central Ion Cyclotron Resonance Heating was applied with $\mathrm{P}_{\mathrm{ICRH}} \approx 1.5 \mathrm{MW}$ in dipole configuration at a frequency of $\mathrm{f}_{\mathrm{ICRH}}=42 \mathrm{MHz}$ to avoid density profile peaking and impurity accumulation in the core plasma. To stabilize the ELM frequency a small gas puff of $\Gamma_{\text {gas }}=3 \times 10^{21} \mathrm{D} / \mathrm{s}$ was used. This resulted in a stable initial pre-pellet phase with good confinement $\left(\mathrm{H}_{98} \approx 1\right)$ and the desired low $\mathrm{f}_{\mathrm{ELM}}=7 \mathrm{~Hz}$. Pedestal top electron densities and temperatures were about $6 \times 10^{19} \mathrm{~m}^{-3}$ and $1.3 \mathrm{keV}$, respectively. Into this initial plasma the $10 \mathrm{~Hz}$ pacing sequence was launched, producing strong fuelling (nominal $\Gamma_{\text {Pellet }}=2.2 \times 10^{22} \mathrm{D} / \mathrm{s}$, the real value estimated is about 1 $\times 10^{22} \mathrm{D} / \mathrm{s}$ ) accompanied by considerable plasma cooling. To compensate for the pellet induced convective losses $\mathrm{P}_{\mathrm{NI}}$ was increased to $16 \mathrm{MW}$ in the final phase of the pellet sequence to recover the initial plasma energy intending to restore at least the initial pressure profile. 


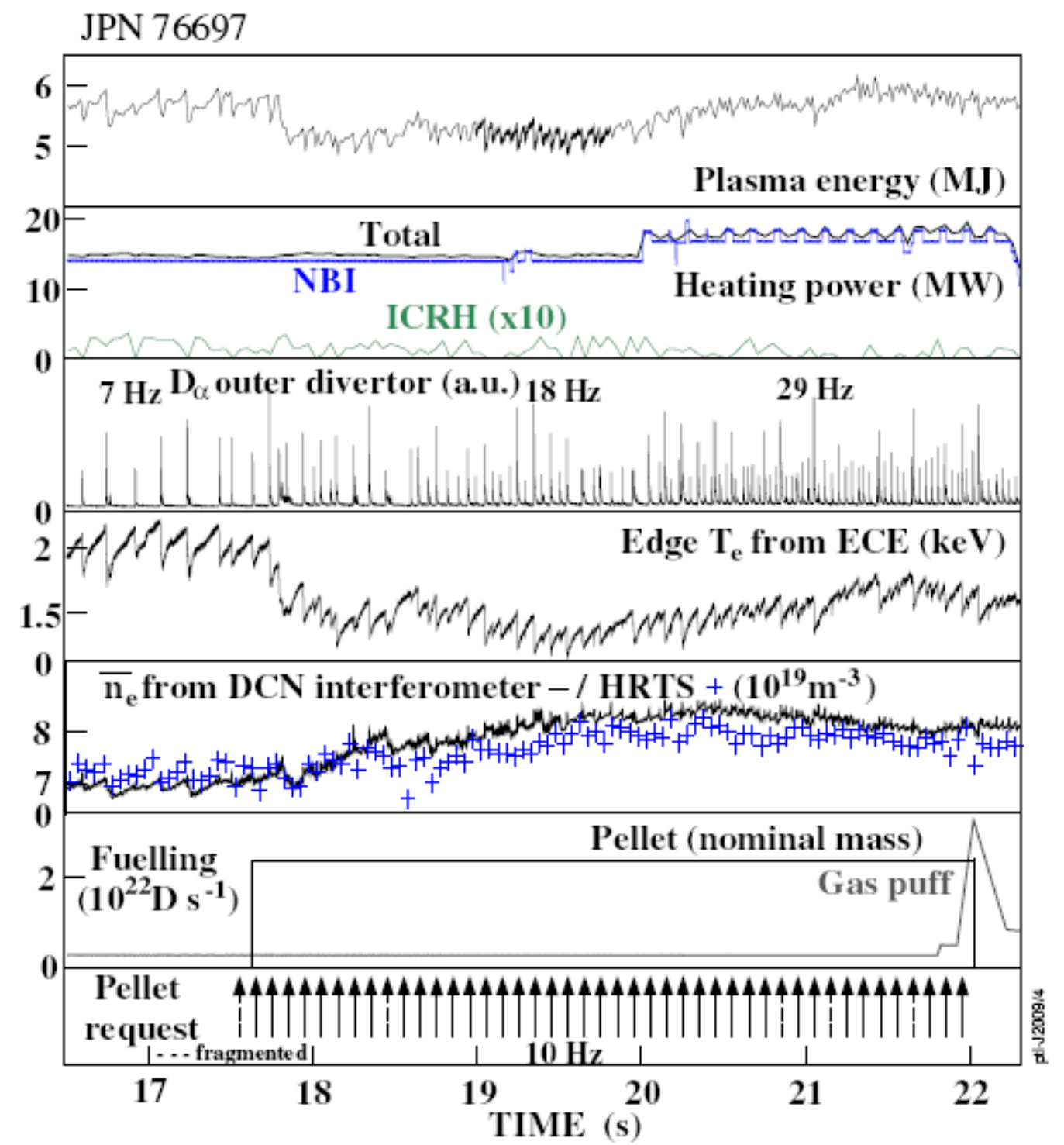

FIG 5.: Pellets injected at $10 \mathrm{~Hz}$ rate during a typical type-I ELMy H-mode ITER baseline scenario. Low fuelling and an accordingly adjusted heating power establish a $7 \mathrm{~Hz}$ initial ELM frequency. Due to the high confinement, fuelling pellets cause significant fuelling resulting in enhanced ELM frequency. During the pellet phase, $f_{E L M}^{0}$ is increased by a factor of 4 while the triggered and spontaneous ELMs are mixed.

Pellet related fuelling resulted in significantly higher densities (about $15 \%$ increase) and lower temperatures in particular at the edge, increasing the spontaneous ELM rate as well. Such an increase in ELM frequency is also observed for gas puff induced density enhancements [13]. The combination of pacing and fuelling drives $f_{\mathrm{ELM}}$ up to about $18 \mathrm{~Hz}$ into a phase composed of triggered and spontaneous ELMs. During this first pacing phase, plasma energy and hence confinement is reduced, attributed to the additional convective losses introduced by the pellet particle flux. When compensating for this loss by increasing $\mathrm{P}_{\mathrm{NI}}$ during the final pacing phase the plasma stored energy was restored. Increasing the power flux crossing the separatrix further raises $\mathrm{f}_{\mathrm{ELM}}$ to $29 \mathrm{~Hz}$ with still $10 \mathrm{~Hz}$ contributed by triggered ELMs, leading to a larger fraction of spontaneous ELMs. Extracting the paced contribution, the spontaneous ELM frequency is almost kept from the initial phase $(7 \mathrm{~Hz})$ into the first pacing phase $(8 \mathrm{~Hz})$ indicating effects from fuelling (expected to raise $\mathrm{f}_{\mathrm{ELM}}$ ) and reduced confinement (expected to lower $\mathrm{f}_{\mathrm{ELM}}$ ) almost chancel. For the final phase the 
(expected) rise of the spontaneous contribution to $19 \mathrm{~Hz}$ is only due to increased heating power since fuelling was kept. Reckoning the evolution of $\mathrm{f}_{\mathrm{ELM}}$ during the pellet phases the attempt for full pacing by gaining full control of the ELMs has fallen short, because fuelling side effects resulted in a similar or even stronger change of the ELM behavior. And during the final phase with higher auxiliary heating power $f_{\mathrm{ELM}}$ raised even further beyond $\mathrm{f}_{\mathrm{PEL}}$. Nevertheless, the experiment allows for direct comparison of spontaneous and triggered ELMs under identical plasma parameters, which will be discussed in the next chapter. These experimental results hint that the smaller nominal $\left(\Gamma_{\text {Pellet }}=0.6 \times 10^{22} \mathrm{D} / \mathrm{s}\right)$ and more frequent $(60 \mathrm{~Hz})$ pacing pellets will fulfill the initial physics requirements to the system. Thus, a HFPI pacing section reaching the nominal performance is expected to be capable of demonstrating a tenfold enhancement of ELM frequency by pellet pacing in an ITER relevant scenario.

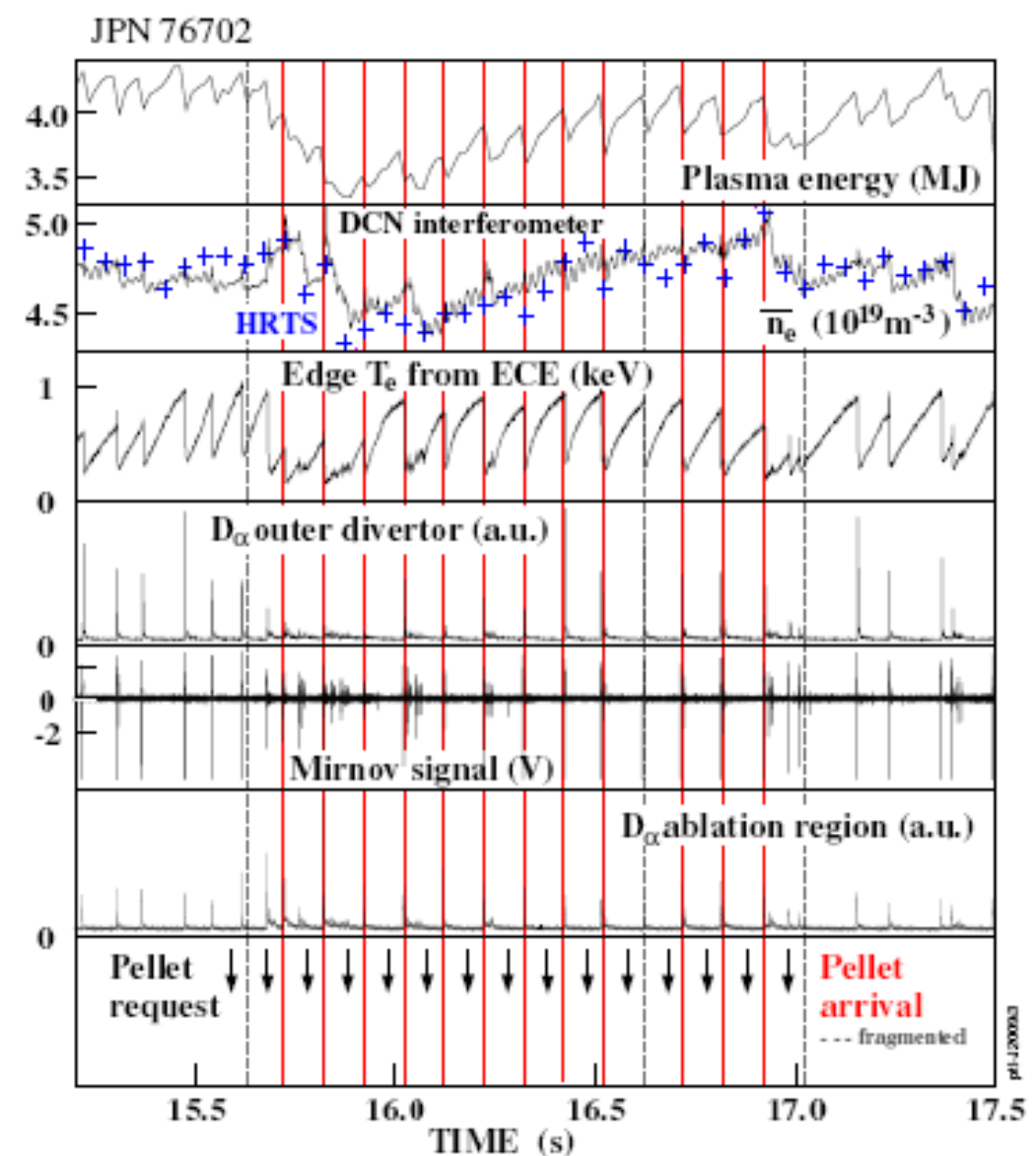

FIG. 6: Confirmation of the ELM pellet pacing technique at JET. Pellets injected at $10 \mathrm{~Hz}$ rate establish a full control of the ELM frequency. Using the fuelling system results in significant density enhancement and convective losses reducing the confinement.

In order to overcome the side effect of fuelling, without the option to bring the pellet particle flux further down, the particle confinement had to be deteriorated. This was done by taking a less shaped configuration at reduced current. The best attempt is shown in figure 6 , again with $3 \mathrm{~mm}$ (nominal $2.2 \times 10^{21} \mathrm{D}$ ) pellets launched at $10 \mathrm{~Hz}$, but at about $200 \mathrm{~m} / \mathrm{s}$ speed. Pellet arrival times at the separatrix were calculated taking the actual pellet speed and the timing when passing the microwave cavity $\mu \mathrm{L}$, the approach being validated for pellets showing a clear impact on the interferometer channels. Further attempts to establish an even higher pellet frequency $\mathrm{f}_{\mathrm{Pel}}$ and do pacing with $\mathrm{f}_{\mathrm{Pel}}>\mathrm{f}_{\mathrm{ELM}}^{0}$ failed as the extrusion limit obviously was hit. The plasma was run at $\mathrm{I}_{\mathrm{P}}=2.0 \mathrm{MA}, \mathrm{B}_{\mathrm{t}}=2.3 \mathrm{~T}\left(\mathrm{q}_{95}=3.8\right)$ with $\delta_{\mathrm{u}}=0.30, \delta_{1}=0.24, \kappa=$ 1.70 and $\mathrm{V}_{\mathrm{p}}=80 \mathrm{~m}^{3}$. The only auxiliary heating was $\mathrm{P}_{\mathrm{NI}}=11 \mathrm{MW}$, no gas puffing. This 
resulted in a stable initial pre-pellet phase with reasonable confinement $\left(\mathrm{H}_{98} \approx 1.1\right)$ and $\mathrm{f}_{\text {ELM }}^{0}$ $\approx 10 \mathrm{~Hz}$, pedestal top electron densities and temperatures were about $4 \times 10^{19} \mathrm{~m}^{-3}$ and 1.1 $\mathrm{keV}$, respectively. In this case the pellets did not cause a significant long lasting alteration of the initial plasma parameters once the perturbation was imposed by switching on the pellet train. ELM pacing could not increase the initial ELM frequency since $\mathrm{f}_{\mathrm{Pel}}=10 \mathrm{~Hz} \approx \mathrm{f}_{\text {ELM }}^{0}$ but it becomes clear the ELMs are generally synchronized to the pellet injection. All sound pellets arriving in the plasma do trigger ELMs and almost all ELMs appearing during the pellet sequence were triggered by pellets - with the exception of one ELM occurring spontaneously on time despite the arrival of a fragmented pellet and one additional spontaneous ELM incidence just about $10 \mathrm{~ms}$ before the next pellet triggers another ELM. Although the ELM frequency could not be increased by the pellets for the obvious reason ELM evolution got locked to the pellet appearance and the pacing attempt achieved control over the ELM evolution. Thus ELM synchronization by pellets was established indicating the pacing technique is in principle also applicable in a large size tokamak like JET.

\section{ELM trigger physics investigations}

Despite of tight technical restrictions to establish steady state conditions with the ELMs under full control by pellet pacing, a wide range of investigations became possible by using any pellet arriving in the plasma to understand the physics underlying pellet triggering of ELMs. In comparison to their spontaneous counterparts, the dynamics of triggered ELMs was studied. This comprises the analysis of the onset speed and mode structure of the magnetic perturbation, the temporal and spatial evolution of the filaments and the power loads of a single ELM but also the temporal evolution of the ELMing behaviour (regular ELMs or burst, ELM types). In a perturbative regime with pellets reaching the plasma at lower rates than the spontaneous ELM frequency they thereby impact on the natural ELM cycle at the time of pellet arrival. In these experiments mostly large pellets injected at low frequency were used for probing ELM triggering or for fuelling purposes. To characterize pellet triggered events neighboring spontaneous ELMs were taken as reference case. A wide range of different scenarios were explored in this way.

Use was also made of the first pacing pellets from a size regime far below the usual fuelling level, approaching values expected for optimized pacing. By this way a possibility arose to find out the threshold conditions for ELM triggering. In cases no ELM was trigged the pure pellet plasmoid induced perturbation was investigated. Since for such experiments every pellet performs an individual test, delivery reliability is not as important as for steady state pacing approaches.

It should be noted that results from these sporadic triggered ELMs investigations cannot be directly extrapolated to the steady state pellet pacing regime. Experiments at ASDEX Upgrade achieving full ELM control at about twice the initial ELM frequency showed that pellet pacing instantly establishes the increased ELM frequency. However the adaptation of ELM size to the higher frequency takes about $100 \mathrm{~ms}$ [5]. Triggered ELMs still do cause the same energy losses as the preceding spontaneous ones at the onset of the pellet train and only gradually approach the final mitigated steady state level while some reduction in energy confinement takes place (effect mainly attributed to the convective losses added by the pellets). Hence, ELM losses and ELM induced power loads can differ quite significantly for sporadic and paced ELMs like observed in ASDEX Upgrade where sporadic ELMs show losses and power loads more like spontaneous events for the same plasma conditions while paced ELMs do look like spontaneous ELMs occurring at the same rate (but at different plasma conditions) [5]. Nevertheless such experiments are very valuable to investigate processes underlying the ELM trigger mechanism caused by the pellets. 


\subsection{Trigger investigations in different scenarios}

Fuelling size pellets were injected mostly from the LFS either accessorily as single pellets or short trains to probe the impact on the ELM behavior in a specific target plasma or on demand in persistent sequences for fuelling purposes. These pellets oversized for pacing requirements can have a significant impact on the plasma conditions and cause a strong transient enhancement of particle and energy flux through the edge pedestal region. Hence, care must be taken for the interpretation of such experiments since the excessive mass deposition close to the edge does not necessarily match plasma conditions achieved in the final pacing approach. In all the investigated scenarios a similar behaviour was observed with the triggered ELMs showing only small differences and some additional features with respect to their spontaneous counterparts. In this section three kind of scenarios will be analyzed: a regime with enhanced field ripple, with resonant magnetic perturbation and with compound ELMs.

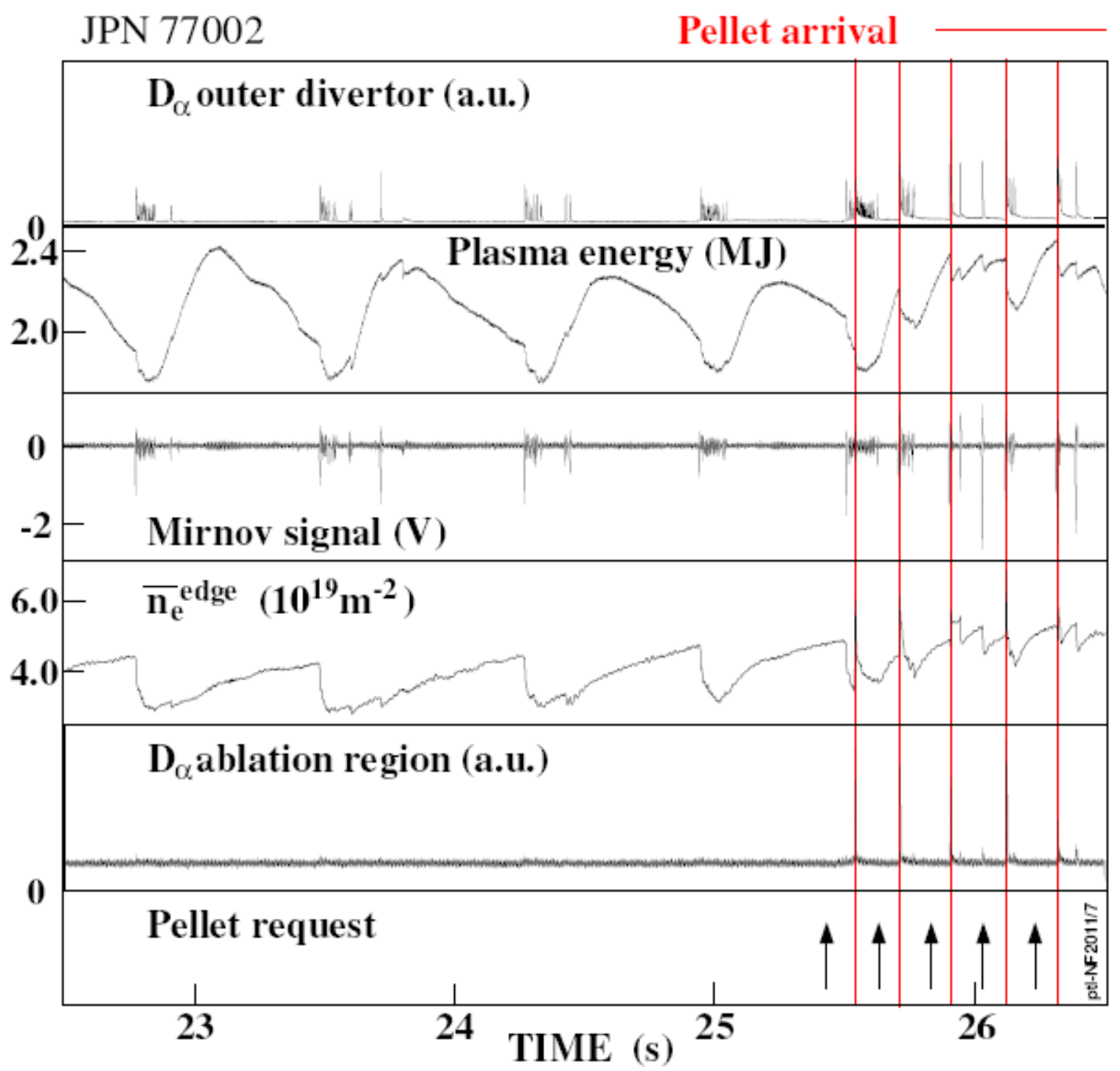

FIG. 7: Pellets impact in plasma with changed ELMing behaviour by increased toroidal field ripple, in this case $\delta_{B T}=0.75 \%$. Pellets launched from the LFS in the final phase to probe the impact on the ELM behavior. Pellets do not create new specific ELM patterns but were able to influence the ELM burst cycles.

\subsubsection{Pellets in toroidal field ripple scan experiments}

The ELM behavior can be strongly affected by the toroidal field (TF) ripple [14]. Defined as $\delta_{\mathrm{BT}}=(\mathrm{Bmax}-\mathrm{Bmin}) /(\mathrm{Bmax}+\mathrm{Bmin})$ the $\mathrm{TF}$ ripple amplitude quantifies the period variation of the TF from its nominal value caused by the finite number and toroidal extension of the TF 
coils. It is possible to vary the TF ripple amplitude at JET on demand and increase it from the nominal value of $0.08 \%$ (at the separatrix outboard mid-plane) up to $3 \%$. In order to investigate the impact on the confinement the ripple was increased in steps from pulse to pulse beginning with an type-I ELM H-mode reference discharge established at a $\delta_{\mathrm{BT}}$ of $0.08 \%$. Ripple scans (experimental details to be found in [14]) with $\delta_{\mathrm{BT}}$ increased up to $1 \%$ showed that ELMs are becoming irregular, the discharge being composed from ELM bursts and long ELM-free phases although the power across the separatrix remains approximately constant. Parasitic pellets did not alter this basic ELM behavior. In the regime with low ripple and regular type-I ELMs the arriving pellets just trigger an additional type-I ELM. Regimes with larger ripple and hence with irregular ELMs are characterized by ELM free phases lasting up to about $0.5 \mathrm{~s}$ interrupted by ELM bursts lasting a few hundred of ms. This interplay of ELM-free and ELMing phases caused also cycles of strong plasma energy variations. These ELMs are composed usually from a strong initial ELM followed by a train of weaker ELMs. Pellets arriving during an ELM-free phase were found to trigger either a singular strong ELM (such events are observed sometimes spontaneously as well) or, more likely the initial strong ELM is followed by a burst of weaker ELMs. A pellet arriving within a burst already ongoing triggers just another weak ELM within this burst. Pellets did not create new specific ELM patterns but were able to influence the cycles. By initiating additional ELM bursts and hence shortening the ELM-free phases pellets were able to moderate the strong variations of the plasma energy. An example for a target discharge with $\delta_{\mathrm{BT}}=0.75 \%$ is shown in figure 7 , here a few fuelling size pellets were launched from the LFS in the final phase to probe the impact on the ELM behavior.

\subsubsection{Compound ELM phases}

A similar behavior was found in the regime of "compound ELMs" [15], observed typically for baseline H-mode scenarios (at nominal ripple) for heating power levels close above the threshold for entering a stable persistent type-I ELMy H-mode. As shown in part A of figure 8, in such cases a sequence of ELM bursts and ELM-free phases appear spontaneously, causing a cyclic variation of the plasma energy similarly to the H-mode discharges with strong toroidal field ripple. The initial strong ELM (regarded as triggered either by a pressuredriven ballooning mode or by a current-driven peeling mode [15]) ejects a significant amount of particles and energy (average loss about $400 \mathrm{~kJ}, \approx 6 \%$ of the plasma energy at the onset) from the plasma and causes a strong drop in both edge density and temperature. After this initiatory event the edge transport barrier remains obviously weakened as the plasma energy decreases for about $30 \mathrm{~ms}$. Incipient recovery is interrupted by further abrupt, but less drastic losses caused by a burst of ELMs (each regarded as triggered by a current-driven peeling mode [15]) weaker than the initial one. Persistent plasma recovery only sets in during the following ELM-free phase. Approaching finally its stability limit, the edge collapses again and the onset of the next initial strong ELM concludes the compound cycle. The impact of a pellet perturbing this spontaneous cycle at different stages is shown in parts B, C and D of figure 8. Any pellet arriving during the ELM-free recovery phase triggers an initial ELM followed by a burst of weaker ELMs despite the plasma edge is not yet recovered to a state where a spontaneous ELM would be due. Arriving within an ongoing burst, the pellet just triggers another weak intra-burst ELM. The intensity of the triggered ELM with respect to power flux to the divertor as measured by thermography and initiated plasma energy drop is also an interesting issue: Pellets arriving late in the ELM cycle are causing ELMs with higher intensity (part B) than pellets releasing an ELM burst just after the previous (spontaneous) one has ended (part C). 


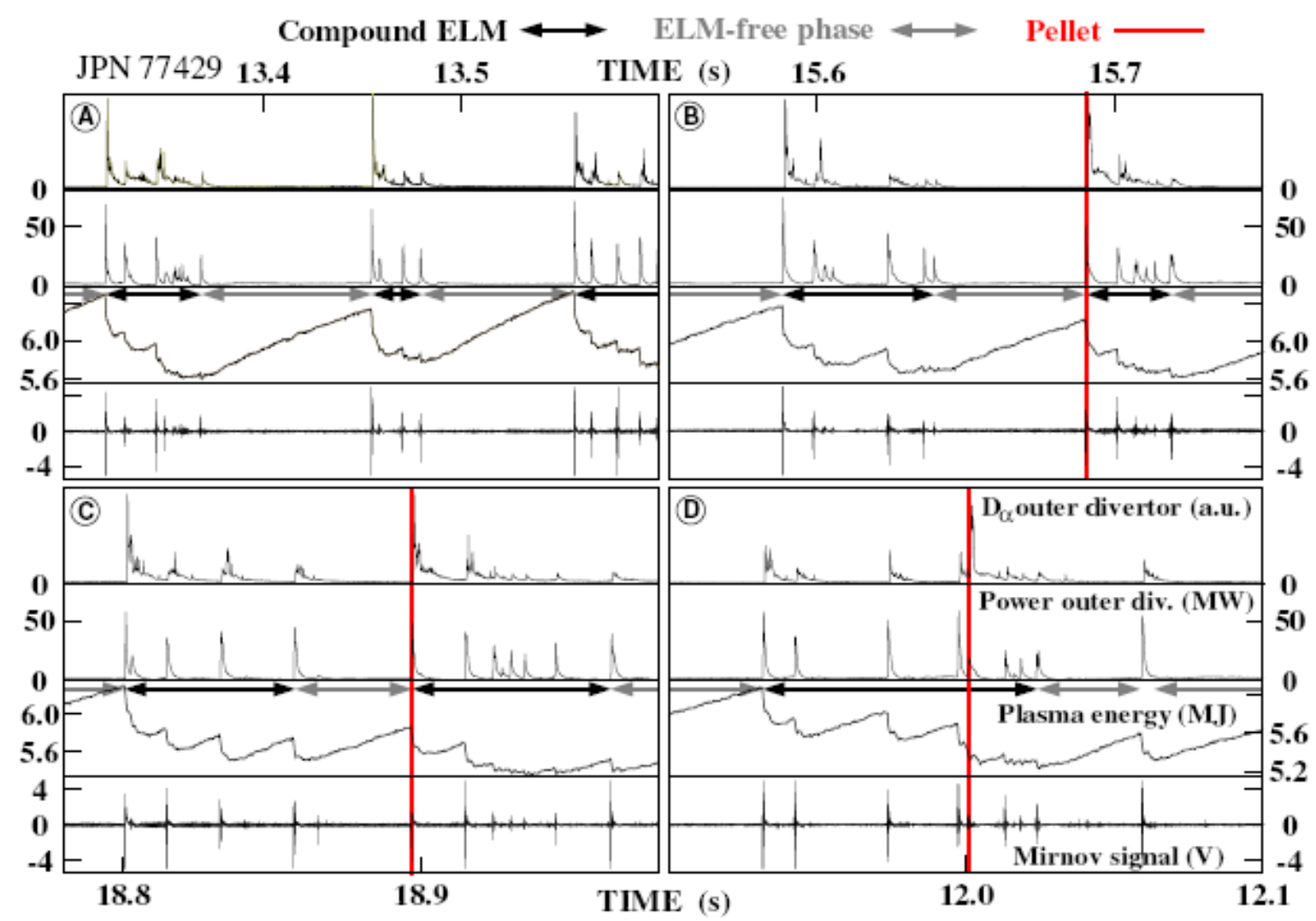

FIG. 8: Pellets impacting at different phases of a compound ELM cycle. The unperturbed evolution is shown in part A. Pellets arriving during the ELM-free cycle trigger an entire ELM burst despite the plasma did yet not fully recover, late triggering (part $B$ ) results in a stronger ELM compared to early triggering (part $C$ ). Arriving within an ongoing burst, the pellet just triggers another weak intra-burst ELM. Please note part A has a different time scale compared to $B, C$, and $D$.

\subsubsection{ELM mitigation phases by external magnetic perturbation}

ELM mitigation experiments with external magnetic perturbation field (EMPF) induced by the error field correction coils EFCCs showed that the frequency and the amplitude of type-I ELMs can be actively controlled by applying $n=1,2$ EMPF [16]. A resonant EMPF can lead to a complete elimination of type-I ELMs and the associated impulsive particle and heat transport avoiding the dangerous transient power load peak rather than making them just more frequent and thereby smaller [17]. At JET a $n=1$ EMPF was found capable to raise the ELM frequency by a factor of 4, while the energy loss per ELM drops accordingly below the noise level of the diamagnetic measurement. Application of EMPF here lead to a density pump out resulting in an unfavorable reduction of the density. Attempts were made to recover the density reduction e.g. by applying additional pellet fuelling [18]. A distinct sequence from such an approach is displayed in figure 9, showing a phase with ELM mitigation enforced by EMPF and ongoing pellet fuelling recovering the initial density level. Arriving pellets trigger ELMs inducing similar MHD activity like the spontaneous ELMs during this phase, as detected by the Mirnov coils. The magnitude of the ELM induced energy losses are correlated to the pellet size. Smaller pellets arriving in the plasma - evidently resulting in a small increase of the edge density - cause energy losses of about the same amount as single spontaneous ELM events. Larger pellets evoking a larger impact on the edge density enforce a significantly stronger energy loss. Here, triggered ELMs are longer and stronger compared to the spontaneous ones, in some cases even an ELM cascade is released. This behavior was observed already in ASDEX Upgrade and is attributed to an enhanced particle flux from the 
plasma edge and hence increased convective losses [19, 20]. In such cases it cannot be distinguished whether the ELM is caused by the direct pellet impact or if it is a secondary effect resulting from the pellet increased density causing a transiently enhanced power flux through the edge. Pellets are causing convective losses which might result in an enhancement of the ELM induced energy loss. However, this effect diminishes with pellet size until for sufficiently small pellets triggered ELMs become alike spontaneous ones. Hence, pellets with a size adopted for the pacing purpose do only trigger ELMs showing the typical features of the originally established ELM regime.

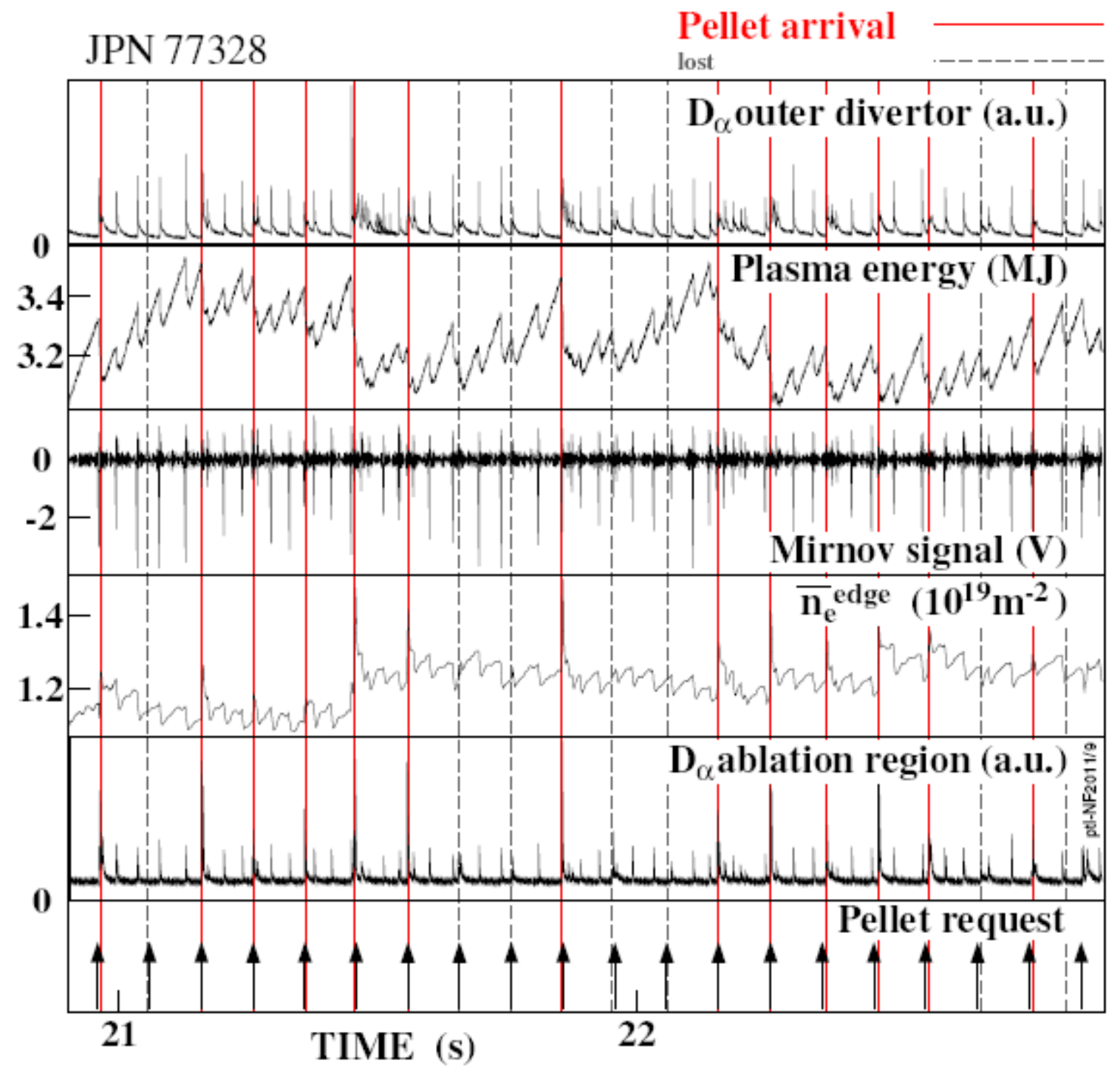

FIG. 9: Pellets impact in a phase of ELM mitigation by external magnetic perturbation fields. Pellets are applied for compensation the density pump out. Large pellets from the LFS cognizable by the large impact on the edge density result in enhanced ELM losses and eventually provoke ELM cascades. Small pellets as indicated by smaller density hub trigger ELMs very likely their spontaneous counterparts.

In consequence this is a strong indication that pellet pacing is compatible with all regimes investigated so far and in particular with other control approaches as EMPF or in configurations with enhanced toroidal field ripple. As it was earlier shown at AUG pellet enforced ELM sustainment is compatible with scenarios where the power flux through the 
plasma edge is controlled by additional impurity radiation [6]. Application of oversized or fuelling size pellets can result in an altered ELM behavior but essentially because they are also changing the plasma parameters in the edge responsible for the spontaneous ELM behavior.

\subsection{Dynamics of triggered ELMs}

For the well established type-I ELM regimes it is found that once the ELM onset is initiated by the pellet, its further evolution is very similar to a spontaneous one. Hence in the type-I ELM regime triggered ELMs basically follow the ELM behavior impressed by the target plasma conditions. As most pellet experiments were performed in this operational regime, there are data available from well matched discharges with and without pellet injection and also from steady state phases with both triggered and spontaneous ELMs.

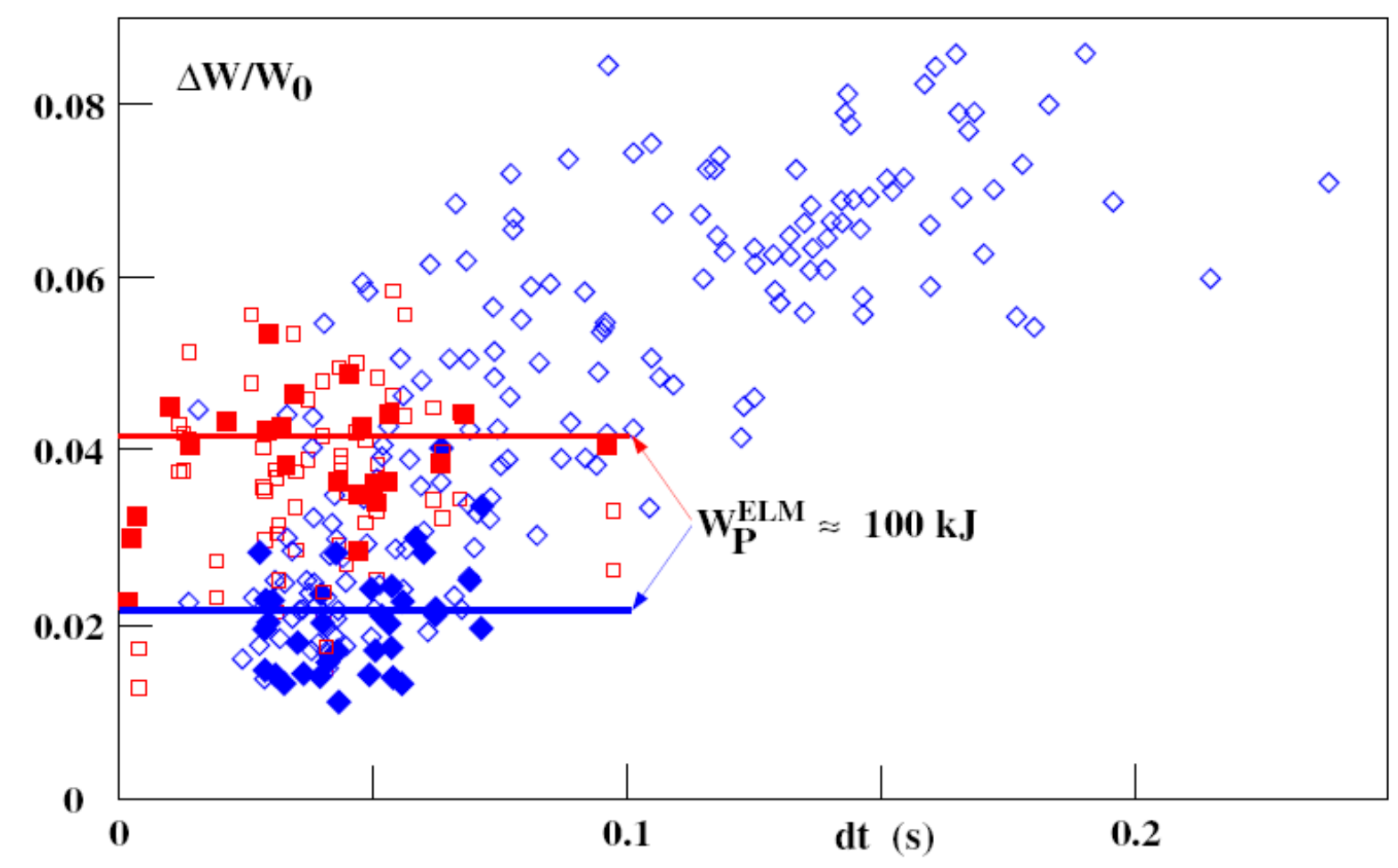

FIG. 10: ELM induced plasma energy losses normalized to the plasma energy content at the ELM onset versus time elapsed since the previous ELM. Data for spontaneous (blue) and pellet triggered (red) ELMs from different discharges with identical scenario. Two dedicated ensembles are taken from a single steady state phase with both types interlocked (filled squares). On average, triggered ELMs of this ensemble show extra losses of about $100 \mathrm{~kJ}$. This is in good agreement with estimated additional convectional losses caused by these fuelling size pellets.

\subsubsection{MHD activity and convection losses}

The similarity of spontaneous and triggered ELMs has already been confirmed by studies comparing magnetic perturbations spectra and the toroidal mode number (n) applying a wavelet analysis to provide good time resolution of short-lived coherent modes [21]. In all configurations investigated triggered events showed a coherent mode structure, indicating that pellets can trigger an MHD event basically in every background plasma. Both for spontaneous and triggered ELMs two components with distinct frequencies and toroidal mode numbers could be detected: one at low frequency and low mode number, typically $n=1$, and another one at higher frequencies, but with typically negative $n$ values [21]. In the high confinement 
type-I ELM regime both triggered and spontaneous ELM starts from a magnetic perturbation with low toroidal mode numbers, then $n$ increases to its maxima before the main ELM burst. The value of $n$ at which the spectrum of triggered ELM peaks saturates was approximately $n=6$, while for spontaneous type-I ELMs this value may be higher up to about 10 within 0.3 $\mathrm{ms}$ before the ELM crash [22].

From fuelling and pacing experiments performed at ASDEX Upgrade it was found the dominant contribution to pellet induced confinement losses both in the inter- and intra-ELM phases are the additional convective losses resulting from the extra particle flux imposed by the pellets [19]. The total collateral energy loss per pellet $W_{P}$ can be estimated assuming thermalization of pellet particles to an average temperature $<\mathrm{T}>$ as $\mathrm{W}_{\mathrm{P}}=3 \mathrm{k}_{\mathrm{B}} \mathrm{N}_{\mathrm{P}}<\mathrm{T}>$ where $\mathrm{Np}$ is the pellet particle inventory. For the additional convective losses of a pellet triggered ELM $_{\mathrm{P}}{ }^{\mathrm{ELM}}=3 \mathrm{k}_{\mathrm{B}} \mathrm{N}_{\mathrm{P}}{ }^{\mathrm{ELM}}<\mathrm{T}>, \mathrm{N}_{\mathrm{P}}{ }^{\mathrm{ELM}}$ being the amount of pellet particles expelled during the ELM. Figure 10 shows the relative energy losses ( $\triangle \mathrm{W}$ plasma energy loss during the ELM, $\mathrm{W}_{0}$ plasma energy at the onset of the ELM event) both for spontaneous (blue) and triggered (red) ELMs using fuelling size pellets from different discharges. Only phases with virtually identical plasma configuration were taken and data were ordered with respect to the time $\mathrm{dt}$ elapsed since the previous ELM. Two dedicated ensembles (filled squares) of triggered and spontaneous ELMs are taken from a single persistent steady state phase were both ELM types occur interlocked. Data of spontaneous ELMs do clearly show the expected correlation of ELM size and frequency, but also reflect the strong scatter found for different individual ELMs even under presumably steady conditions making it impossible to derive an unambiguously precise conclusion from the comparison of two single ELM events. Instead, averaging over the two dedicated ensembles reveals that triggered ELMs are ordinarily larger with respect to spontaneous ones. For the given plasma conditions the extra losses $\mathrm{W}_{\mathrm{P}}{ }^{\mathrm{ELM}}$ are about $100 \mathrm{~kJ}$. Estimating $\mathrm{N}_{\mathrm{P}}{ }^{\mathrm{ELM}} \approx 3 \times 10^{20} \mathrm{D}$ from the LIDAR and interferometer density measurements results in $\langle\mathrm{T}>$ of $500 \mathrm{eV}$. This fits quite well to the magnitude of expected convective losses since the (electron) pedestal top temperature of the target plasma was about $1 \mathrm{keV}$ and ELM induced losses of pellet deposited particles are anticipated coming predominantly from the edge barrier region.

For cases where pacing size pellets were used for ELM triggering no significant difference was found for the ELM induced losses with respect to the ones by spontaneous ELMs. However, only very few data are available yet going along with a weak statistical relevance. Clearly further dedicated investigations are required in order to sound out if pellet imposed convective losses really vanish with pellet size adopted for pacing purpose.

\subsubsection{Ablation cloud and ELM filament evolution}

Trigger investigations so far have shown obviously a pellet can trigger the onset of an ELM due to a strong seed perturbation already under conditions where the unperturbed plasma edge is still stable. Pellets do trigger ELMs of the type typical for the according plasma regime or phase (type-I or type-III, initiating a new ELM burst or adding just another small ELM to a burst) but can show some difference compared to their spontaneous counterparts. Such differences are e.g. an accelerated onset of the MHD activity or the absence of precursors [23] and can be explained by the forced ELM onset or due to the fuelling coming with the pellet. Trigger experiments performed in this study further support this interpretation.

The almost prompt (time scale in the order of about $100 \mu \mathrm{s}$ ) trigger was explained by the poloidally and toroidally asymmetric plasma pressure perturbation induced by the pellet cloud. Along the field lines and ultimately within the whole flux surface, a strong pressure enhancement concentrates in the small region filled by the newly ablated material causing in the same time a general pressure reduction over the rest of the surface. This pressure perturbation, as the helical, finite amplitude seed of a pressure driven mode, was attributed as 
the cause for the ELM triggering [24]. Recently, this qualitative explanation was confirmed by modelling a pressure driven mode for a non-equilibrium situation with non-constant pressures on a flux surface applying the non-linear MHD code JOREK with the pellet represented by a large source term in the density equation $[25,26]$. A pellet modeled as a large amplitude (initial state of density perturbation has a maximum amplitude of 25 times the background density, total added number of particles $6 \%$ of the initial plasma particle content), localized, (initially) static density perturbation imposed on the $\mathrm{H}$ mode pedestal creates a high density plasmoid large enough to drive ballooning type modes forming a single helical structure located at the pellet (plasmoid) position [26]. An investigation envisaging to sound out onset conditions for driving the mode unstable in comparison to real experimental conditions is under way.

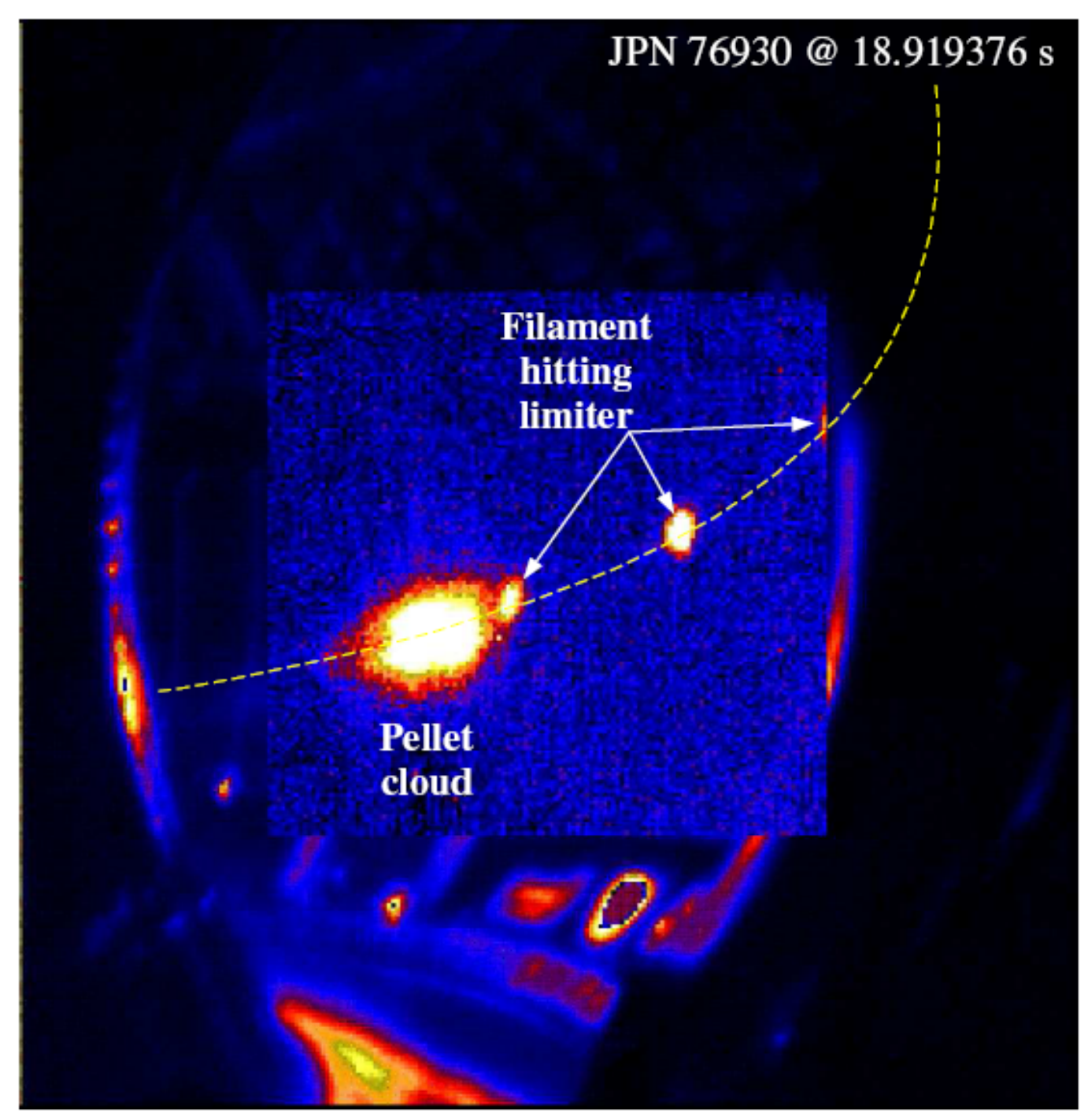

FIG. 11: View of the fast framing camera at the onset time of strong MHD activity for an ELM triggered by a large pellet injected from the LFS. The large slow (56 $\mu$ s resolution) framing image covers the entire camera view while the fast (14 $\mu$ s resolution) framing image only can cap the central part. Bright spots appearing transiently at limiter structures in addition to the dominant pellet ablation cloud are aligned to the estimated field line (dashed line, from EFIT equilibrium reconstruction) starting at the position of the pellet trajectory crossing the separatrix ( $\left.q_{95}=3.6\right)$ [27]. 
Observations are in good agreement with a proposed triggering mechanism assuming the first filament releasing the ELM initiated by the high pressure plasmoid generated by the ablating pellet. Bright spots can be seen on limiter elements located at the outboard wall of the torus by a fast framing camera observing this region in the case of large pellets injected from the LFS as shown in figure 11 [27]. The onset of these spots corresponds to the onset time of strong MHD activity at the edge coming with the ELM. Similar structures are observed at the onset of sufficiently strong spontaneous ELMs. But different to their spontaneous counterparts showing strong spatial scatter for different events, in the pellet triggered case the onset is always observed at the same fixed position coinciding with a field line passing the pellet ablation zone close to the separatrix. It is hence concluded these bright spots are caused by the impact of filaments initiated by the plasmoid created by the pellet close to the separatrix [27].

Further experimental evidence for this single filamentary structure following the field line is provided from measurements of the ELM induced divertor heat loads. It is found that the heat flux footprints of a pellet triggered ELM in some cases show a dominant additional peak. In the discharge investigated large fuelling size pellets $\left(\sim 2 \cdot 10^{21} \mathrm{D}\right)$ have been injected. In the discharges a toroidal magnetic field $\left(\mathrm{B}_{\mathrm{T}}\right)$ scan from $2.2 \mathrm{~T}$ up to $2.8 \mathrm{~T}$ at fixed plasma current of 2.0MA was performed. Energy deposition profiles from the first $400 \mu$ s after the peak power load are compared for spontaneous and pellet induced ELMs at varying $\mathrm{B}_{\mathrm{T}}$. Spontaneous ELMs show profiles with one main peak whose location is independent of $\mathrm{B}_{\mathrm{T}}$. On the other hand pellet induced ELMs are associated with a relatively dominant peak, but this has a radial location scaling with $\mathrm{B}_{\mathrm{T}}$. Field line tracing based on the pre-ELM magnetic equilibrium reconstruction was used to predict the target deposition location in the toroidal position of the IR view of SOL filaments crossing the pellet injection trajectory. Here, throughout the full $\mathrm{B}_{\mathrm{T}}$ scan excellent quantitative agreement was found between this calculations and the location of the experimentally measured peak heat flux associated with pellet induced ELMs [28].

However, these observations of filaments in the mid plane and the divertor were made only when using large pellets. In case of small pellets triggering an ELM, no strong filament could be detected by the framing camera while for the divertor thermography $B_{T}$ scan a corresponding experiment has not yet been carried out.

In case of a small pellet not triggering an ELM, the resulting small pressure pulse to the divertor shows the same dynamics as an ELM as is shown in figure 12. The left part displays the evolution of main plasma parameters during an approach of launching pacing size pellets (nominal $1.1 \times 10^{22} \mathrm{D}$ ) at about $160 \mathrm{~m} / \mathrm{s}$ speed from the VHFS into a ITER baseline scenario plasma with high triangularity. A low spontaneous ELM frequency was established to allow for dedicated trigger investigations, since this tiny pellets cause a very weak perturbation. Only 2 out of more than 20 pellets launched reached the plasma in reasonable shape according to dedicated VHFS ablation radiation monitor signal.

Obviously, one pellet triggers an ELM while the other fails to do so. Interestingly, it is the presumably smaller pellet arriving later in the ELM cycle triggering the ELM while the presumably larger one arriving early in the cycle does not. However care has to be taken by comparing the pellet sizes, as no precise pellet size measurement was available. Due to the stable plasma conditions - variations determined by the spontaneous ELM cycle - different events can be compared under almost identical conditions in the phase shown. Three cases are picked from this phase and shown on an expanded time scale on the right part of figure 12 . Case A marks the pellet failing to trigger an ELM; case C the pellet triggering an ELM and B a typical spontaneous ELM (special choice of no relevance). Since the spontaneous ELM (B) and the pellet triggered ELM (C) have in common the onset of the ELM and both pellets have in common the onset of pellet ablation (presumably when crossing the separatrix) time traces of all three events can be ordered altogether taking pellet ablation and ELM onset as 
coinciding time marks (solid grey lines). For all three cases an increase in the radiation from the outer divertor region is observed with very similar timing for this ordering with the radiation magnitude of the pure pellet case (A) much smaller than for the ELM cases (note different scales!). For both the spontaneous and triggered ELM case this radiation event is assigned to the energy losses caused by the ELM mainly carried by the ions with an onset delayed to the ELM at a characteristic time $\tau^{\text {ion }}=\mathrm{L}_{\|} / \mathrm{c}_{\mathrm{S}}$ describing the transport of ions in the scrape off layer (SOL) towards the divertor plates [29]. For the characteristic connection length of the field lines in the SOL we estimate $\mathrm{L}_{\|}=2 \pi \mathrm{q}_{95} \mathrm{R}_{95}=88 \mathrm{~m}$ with $\mathrm{q}_{95}=3.6$ and $\mathrm{R}_{95}$ $=3.9 \mathrm{~m}$. Following [29] and taking $\mathrm{T}_{\mathrm{e}}=\mathrm{T}_{\mathrm{i}}=\mathrm{T}_{\text {ped }}$ with $\mathrm{T}_{\text {ped }}$ the pedestal top temperature - here about $1 \mathrm{keV}$ - the ion sound speed $\mathrm{c}_{\mathrm{S}} \approx 3 \times 10^{5} \mathrm{~m} / \mathrm{s}$ results in $\tau^{\mathrm{in}}{ }_{\|} \approx 0.3 \mathrm{~ms}$. This fits exactly the time delay observed between the peak of ablation radiation and the peak $D_{\alpha}$ radiation in the outer divertor in the pure pellets case while a slightly shorter delay $(0.2-0.25 \mathrm{~ms})$ is observed in case of the pellet triggered ELM. The purely pellet born pressure pulse resulting from parts of the ablation plasmoid structure partially expelled from the plasma obviously follows the same dynamics as the ELM filament.
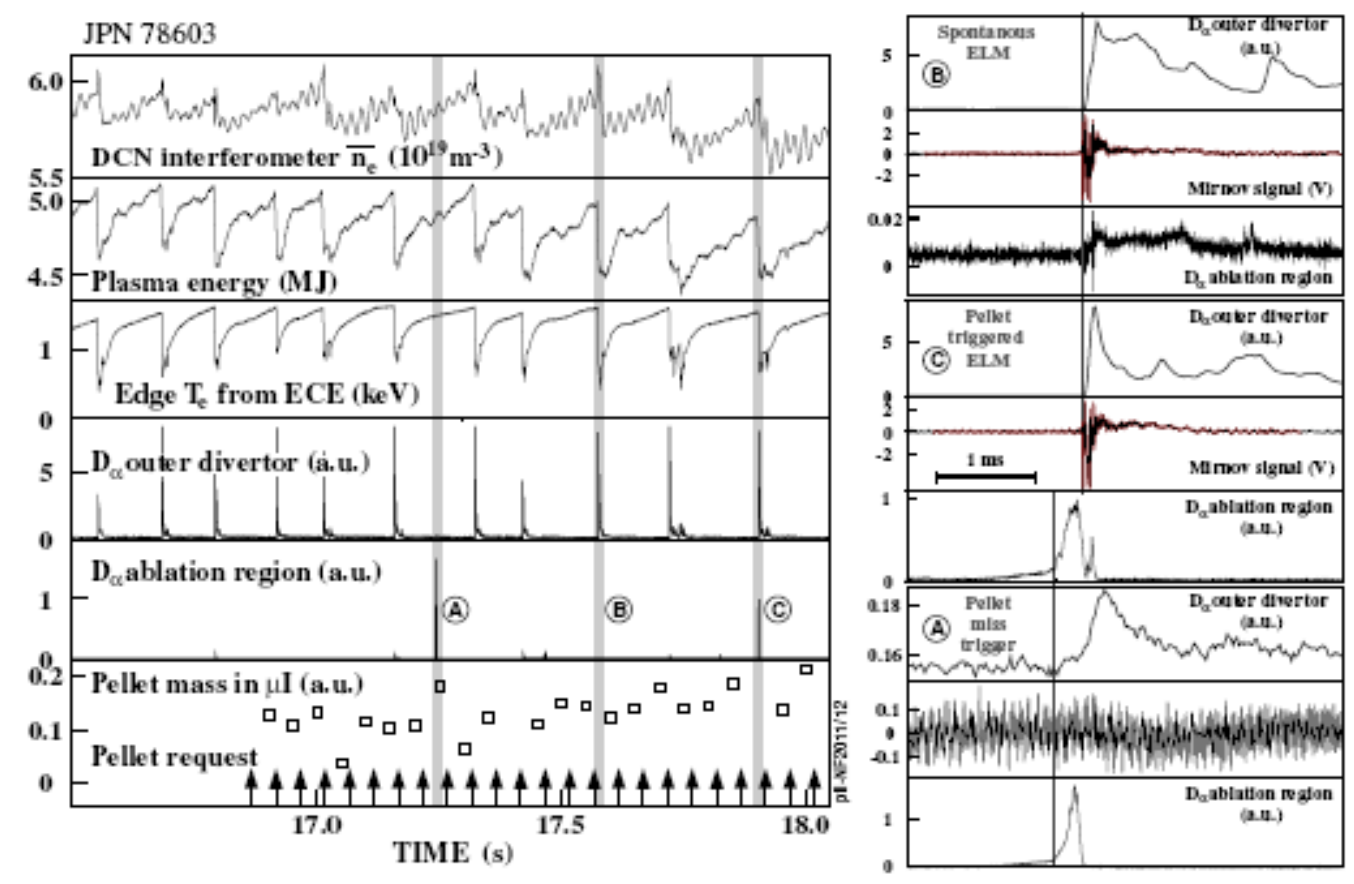

FIG. 12: Left: Small VHFS pellets injected during steady state low frequency type-I ELMing phase; pellet $C$ triggers an ELM while pellet $A$ does not, arbitrary spontaneous ELM B. Right: Marked cases with time scales ordered (full grey lines) by onset of ELM ( $B$ and $C$ ) or pellet ablation ( $A$ and $C$ ). The pressure pulse (indicated by the enhanced $D_{\alpha}$ radiation) resulting from the pure pellet plasmoid arrives in the divertor with the about same delay observed for both the triggered and spontaneous ELM (dashed grey line).

\subsection{Trigger threshold investigations}

It is thought the minimum possible pellet size and hence unavoidable fuelling is determined by the minimum pellet penetration required to establish a sufficient perturbation for the triggering. Using the pacing section of the launcher for the first time investigations to find out the according pellet size became possible. This is shown in the experiment presented in figure 13 where pacing size pellets (nominal $1.1 \times 10^{22} \mathrm{D}$ ) were injected at about $200 \mathrm{~m} / \mathrm{s}$ speed from the LFS into a ITER baseline scenario plasma with high triangularity. Several pellets arrive in the plasma and some clearly do not trigger ELMs. Pellets not triggering ELMs reach 
the plasma in fairly good shape still carrying a significant fraction of the designated mass according to the response of mass measurements along the injection tube and also from the fast framing camera images detecting rather compact cloudlets. The diode based ablation radiation monitor is not optimized for the LFS injection and does not allow for a clear distinction between pellets and ELMs in this pellet size (and quality) domain. Instead, the monitor signal derived from the framing camera is used, providing a fairly good measure of the pellet ablation evolution. Thus definitely it can be concluded injecting pacing size pellets into scenarios where fuelling size pellets achieved reliable triggering showed that some but not all pellets trigger ELMs. Hence it became clear using the pacing settings for the matching plasma parameters pellet size and velocity were in the vicinity of the trigger threshold.

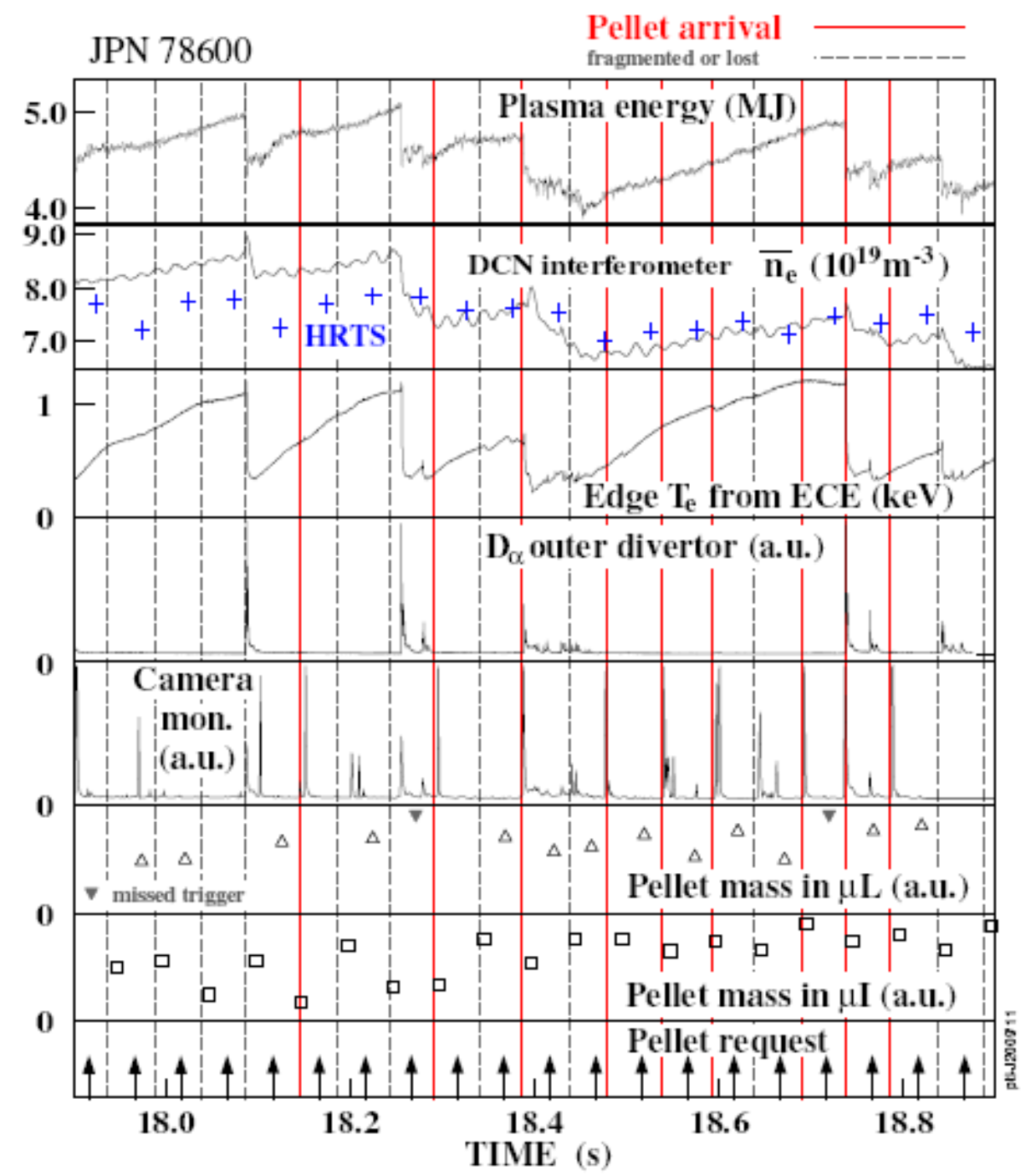

FIG. 13: Small fast LFS pellets injected in a plasma configuration where fuelling size pellets achieved reliable ELM triggering. Some pellets in this size domain do no longer result in an ELM triggered despite arriving presumably still in good shape carrying a significant fraction of the design mass. This can be concluded from the timely transfer through microwave cavities along the tube and the according mass measurements and the fast framing camera images observing pellet ablation.

Since there is a strong mass scatter already within the pellets of a single train launched with a fixed nominal size and speed a dedicated pellet mass scan was not practicable. Pellets that do enter the plasma from the VHFS are clearly seen on the pellet $\mathrm{D}_{\alpha}$ monitor. They can be finely 
correlated in time with $\mathrm{D}_{\alpha}$ from the ELM and a fall in edge SXR emission [23]. Their relative size can be estimated from the signal-level in the $\mathrm{D}_{\alpha}$ monitor. In Figure 14 the peak pellet monitor pulse-height is plotted against the number of pellet requests (only about 20\% reaching the plasma) for a train launched from the VHFS. It is clearly seen that not all pellets can trigger an ELM and that there is a threshold in the pellet size required to trigger an ELM [23].

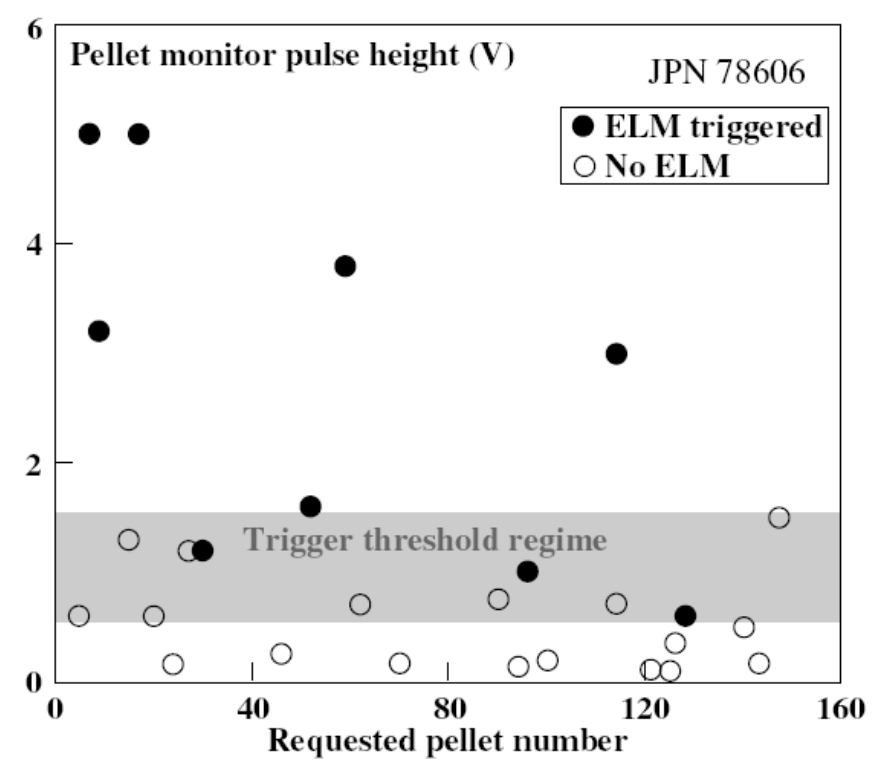

FIG.14: Pellet monitor pulse height against pellet request number for a train launched from the VHFS. Pellets trigger an ELM are shown as dots and typically are larger in size than pellets not causing an ELM. Obviously, there is a mass trigger threshold in the size regime correlating to about $1 \mathrm{~V}$ monitor signal [23].

As pellet mass is one of the candidates responsible for a threshold like behaviour for the ELM trigger, several experimental and modelling activities were performed to find out the minimum pellet mass enough to trigger an ELM. The intensity of the $\mathrm{D}_{\alpha}$ radiation is known to follow the pellet ablation rate in a wide plasma parameter range. Assuming a linear correlation of radiation to ablation and normalizing to the maximum observed pellet size to the maximum monitor response $(5 \mathrm{~V})$ the observed signal threshold of about $1 \mathrm{~V}$ can be converted into a mass threshold. During ELMy H-mode phases these small pellets are not found to produce any net plasma density increase and no impact on any observed plasma parameter was detectable. Thus, pellet injection was continued in same cases using a L-mode plasma phase for monitoring. For L-mode phases showing a more quiescent evolution of edge parameters the pellet impact was emerging compared to the dynamic cyclic changes imposed by the ELMs during H-mode phases.

Applying the JET Integrated Transport Suite of Codes JINTRAC including the pellet ablation and deposition code HPI2, these L-mode phases were modeled [30]. Compared with measurement data to determine the range of effective pellet masses reaching the plasma a maximum value of $4-5 \times 10^{19} \mathrm{D}$ was obtained for the pacing pellets arriving in the plasma. If the $\mathrm{D}_{\alpha}$ pulse height is proportional to pellet mass entering the plasma the trigger threshold is above $\sim 10^{19} \mathrm{D}$, or instead using the total integral $\mathrm{D}_{\alpha}$ emission above $\sim 1.6 \times 10^{19} \mathrm{D}$ [23]. Pellets of this size do - according to modeling [30] and time of flight estimations [23] using the ablation duration - penetrate to at least half of the pedestal width. Figure 15 shows the results of pellet ablation modeling for LFS launch under typical plasma and pellet conditions applied in trigger experiments using pacing size pellets. In the code runs, different particle 
inventories were considered. Obviously, pellets sufficiently large to trigger an ELM penetrate deep into the edge transport barrier or even to the pedestal top. Very shallow pellet penetration (few $\mathrm{mm}$ ) is insufficient for reliable ELM triggering. Caution has to be taken however for further conclusions since this mass threshold is a strong but simple empirical correlation. In order to find out on which parameter the trigger threshold is dependent on, more dedicated investigations with higher precision are required. For example there are indications the pellet arrival time with respect to the evolution of the ELM cycle can play a role with the trigger threshold requirements gradually reducing while the plasma edge recovers after an ELM and the pedestal pressure gradient approaches again its linear stability before the next spontaneous or induced crash.

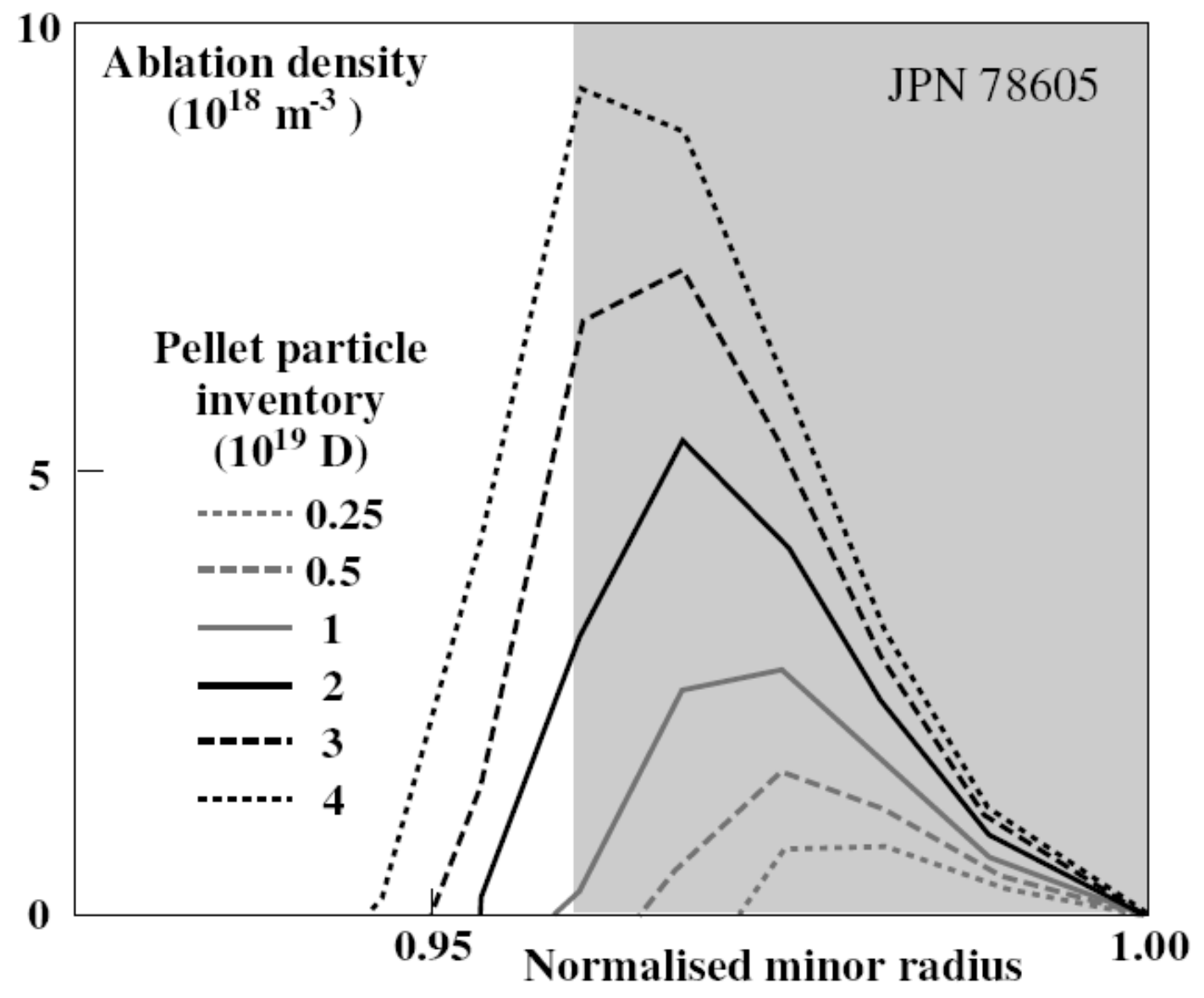

FIG. 15 : Ablation profiles for pellet injection from the LFS under typical plasma conditions used for trigger investigations. The particle inventory of the pellets when ablation set in at the separatrix varied in the size regime of arriving pacing size pellets. For the most probable pellet size at trigger threshold $\left(0.5-2 \times 10^{19}\right.$ D) pellets are expected to penetrate deep into the edge transport barrier region (indicated as grey shaded area).

Finally it should be noted that a more thorough investigation of the impact caused by injection location, i.e. the poloidal launch position, on the trigger threshold is still required. No indication was found in the experiments reported here for a significant difference in the mass threshold, but clearly the resolution needs further improvement. Recent modeling investigations using the JOREK code predict a very similar mass threshold for inboard and outboard launch with a slightly lower threshold for a pellet perturbation imposed by a LFS pellet directly inside the region where the ballooning instability develops [31]. 


\section{Status of the validation of pellet ELM pacing for ITER}

ELM triggering and frequency control by pellet ELM pacing is a robust and reproducible effect, observed in all major tokamaks. Dedicated experiments were carried out in many devices to understand the underlying physics of the ELM triggering by pellets. These investigations cover a wide range of plasma regimes and pellet parameters. There is a high level of consistency in the results of these experiments carried out in different machines, providing a sound experimental basis. It is likely pellet ELM triggering and frequency control will work in ITER for frequencies close above the spontaneous ELM frequency but nothing proves for now the envisaged significant frequency enhancement can be provided. Like any ELM control tool envisaged for ITER pellet pacing has to substantiate its potential to reduce peak power loads below any critical destruction limit under relevant conditions.

The underlying ELM trigger physics is now understood at least qualitatively. The 3D nonlinear MHD code JOREK [26] describes the evolution of the pellet ablation plasmoid into a first ELM filament in agreement with the experimental observations as described here and as it was also recently shown from fast framing camera observation at DIII-D [32]. In case of large pellet injection a separate increased power load zone in the divertor was observed as it was predicted by JOREK simulations. This additional power load zone can probably form a problem in ITER for its toroidal asymmetry might result in a high strongly localized heat flux. Additionally it would impinge at a position not exposed to significant heat fluxes during a spontaneous ELM. On the other hand, it is also possible the effect can act beneficial by dispersing the ELM load. Further investigations applying small pellets are needed since notable effects of pellet born filament so far were only observed for large pellet injection. Pellet ELM triggering on the experimentally observed time scale can also be explained by the linear MHD stability code MARG2D coupled to integrated core and SOL transport codes handling the pellet imposed perturbation [33].

Technical limitations at JET only allowed experiments mostly conducted under conditions still quite far from ITER relevant requirements. Due to the current status, ITER requires mitigation of the ELM power loads calling for a ELM frequency of about $60 \mathrm{~Hz}$. This could be envisaged by enhancing the spontaneous ELM frequency by about a factor of $10-30$ to this value [34]. Increasing the ELM frequency by pellet pacing reduces the mean ELM size and power load. However, according to the required divertor power flux mitigation ITER might require an ELM frequency enhancement even beyond a value prescribed by the relation $\mathrm{W}_{\mathrm{ELM}} \times \mathrm{f}_{\mathrm{ELM}}=$ const.: in AUG pacing experiments the resulting ELM size probability distribution was found slightly broadened, hence ELMs can occur with a size unusually large for the according regime [5]. Moreover, recent investigations have shown the effective ELM energy deposition area depends on the magnitude of the ELM energy loss and increases significantly with $\mathrm{W}_{\text {ELM }}[35]$. This way for spontaneous and even more for triggered ELMs the maximum expected peak heat flux to the divertor decreases more weakly than $1 / \mathrm{f}_{\mathrm{ELM}}$, consequently increasing the ITER requirements for frequency enhancements over the natural ELM frequency to achieve the target ELM density [34].

Pellet pacing at an absolute rate of $83 \mathrm{~Hz}$ has already been demonstrated at AUG [5], hence establishing a sufficiently high pellet rate for ITER does not cause an engineering problem. However a firm demonstration for pacing covering the full request in a ITER like scenario has not been obtained yet. Characteristics of triggered ELMs have been intensely analyzed, usually comparing them with respect to spontaneous events from the same or from similar reference phases. The pacing regime could be established only in few cases, and all experiments were done with rather large pellets. So far there is no experiment yet performed with pacing size pellets reaching the optimal pacing regime. Operational parameters covered by the present experiments do not yet cover ITER requirements, hence the extrapolation to 
ITER parameters has some risk. This applies in particular to the requested frequency enhancement by a factor of around 30 .

At AUG an ELM frequency enhancement by a factor of 2 was demonstrated for full ELM control with the pellet rate $f_{P E L}$ determining the ELM frequency $f_{E L M}$ in a control sequence starting from the initial $\mathrm{f}_{\text {ELM }}^{0}$ into an about $2 \mathrm{~s}$ long phase reaching a value of $\mathrm{f}_{\mathrm{ELM}}=\mathrm{f}_{\mathrm{PEL}} \approx$ $2 \times \mathrm{f}_{\text {ELM }}^{0}$ and returning back to the original $\mathrm{f}_{\text {ELM }}^{0}$ [5]. As reported here, full synchronization of ELMs with injected pellets $\left(\mathrm{f}_{\mathrm{ELM}}^{0}=\mathrm{f}_{\mathrm{PEL}}\right)$ was now achieved also in JET, confirming that pellet pacing works in the largest tokamak as well. An ELM frequency increase accompanied by an according ELM size reduction was achieved for the "ITER baseline" scenario, however here the increase of $f_{\text {ELM }}$ was mainly caused by secondary effects of pellet fuelling changing the power flux through the edge. DIII-D recently demonstrated an enhancement of the initial spontaneous ELM frequency by factor of about 5 once the pellet train is injected, also accompanied by a strong reduction of the ELM size [32]. However it must be mentioned here as well $\mathrm{f}_{\mathrm{ELM}}>\mathrm{f}_{\mathrm{PEL}}$ is observed indicating at least a part of the frequency enhancement must be due to secondary effects although pellets do not show a noticeable impact on the density.

It seems pellet pacing is compatible with other mitigation or control schemes. In several cases where ELM mitigation or avoidance is achieved by another control approach ELMs triggered additionally are found very similar to the spontaneous event characterizing the according phases; in particular the already reduced ELM size can be maintained. Examples are the ELM sustainment by pellet pacing in otherwise unstable discharges with radiative edge cooling at AUG [6], pellet fuelling of phases with reduced ELM size achieved by EMPF at JET [18] and the impact of pellets during phases with the ELM behaviour altered by enhanced field ripple in JET as reported here.

With the new generation of launcher systems designed to deliver small, pacing size pellets it becomes possible to minimize the unwanted particle flux/fuelling associated pacing. However, these new systems are found technologically challenging, since production and transfer of such small pellets (for existing tokamaks pacing pellets should have sizes in the $\mathrm{mm}$ or even sub-mm range) is a difficult task. Optimization of the pellet tool can only be achieved by reducing the pellet mass as far as possible while keeping reliable pacing. In this operational mode it has to be investigated if the impact on confinement reduces as expected, for a convective loss dominated effect held responsible for the observed magnitude of confinement losses, or if another energy loss channel starts to play a significant role. Reduction of pellet mass would also help to reduce gas throughput requirements for the ITER pumping and detritiation system which had already to be upgraded to incorporate pellet pacing requirement [36]. Consequently, to find out the minimum pellet size and best pacing configuration is still a major task.

So the essential question at present is still to find out what are the threshold conditions for pellet ELM triggering obviously strongly connected to a minimum required pellet penetration depth. This topic is currently under investigations at JET with a launcher capable to access the pacing size domain of pellets, even if only single pellet events are available. However, care must be taken in this spurious triggering regime since there is some response time required for the paced ELM to adapt in size to the imposed elevated frequency. This way the results achieved for a single triggered ELM may not necessarily hold for the more ITER relevant pacing regime. Our experiments show a clear size threshold for ELM triggering in the "ITER baseline" configuration with the size limit in regime $1-2 \times 10^{19} \mathrm{D}$, pellets are expected to penetrate to about pedestal top. This is in good agreement with trigger investigations, relying on pellet time of flight determination [37] but also with the recent pellet size variation experiments from ASDEX Upgrade: pellet penetration to at least half, but more likely the full edge barrier width is necessary to trigger an ELM. Somewhat different results are reported from DIII-D where time of flight experiments indicate triggering by some large pellets 
penetrating only a few $\mathrm{mm}$ into the pedestal [7]. However in recent size variation experiments at DIII-D shallow penetrating pellets did not trigger ELMs [32], in agreement with the JET and AUG findings.

Another important question is the location of the injection. The critical size of perturbation for the pellet perturbation necessary for the onset of the instability seems to be very similar for high field side and low field side pellets. Both from modeling and experimental observations no significant difference in the trigger penetration threshold and the dynamics of the triggered ELM is found. Due to drift effects influencing the ablation dynamics and the pellet speed in the plasma [38] at a given launch speed smaller pellets can reach a required penetration for inboard compared to outboard launch. A pellet pacing system able to deliver high speed inboard pellets arriving at the plasma with sufficient reliability and a pellet mass just sufficient to an ELM seems most adequate. On the other hand the strong pellet erosion found in long curved guiding tubes and more severe speed limitation for narrow bend radii penalize inboard launch requiring a more complex guiding system. Furthermore, the favorable drift effect can even result in unwanted fueling. Hence, a dedicated balancing of the different effects have to be taken into account to find out the best poloidal location for injecting pacing pellets. Pellet velocity scatter resulting in a scatter of pellet arrival times at the plasma could cause an additional problem since a prescribed minimum ELM size could require higher pellet launching rates in order to ensure a sufficient pellet rate on plasma. A recent analysis indicates centrifuge launchers show significantly better performance with this respect than blower or gas guns and hence might be the more suitable solution for ITER [39]. Pellet technology in the parameter range requested for pacing in ITER is mature, the designed pellet system in its recently upgraded form [40] allows for flexibility both with respect to possible injection tracks and pellet parameters. Different launchers are going to be installed for different purposes (besides pacing also fuelling and impurity pellet injection are specified pellet tasks) covering a wide pellet parameter range and it seems appropriate this approach can cope with even changing requirements.

\section{Acknowledgements}

This work was supported by EURATOM and carried out within the framework of the European Fusion Development Agreement. The views and opinions expressed herein do not necessarily reflect those of the European Commission.

\section{References}

[1] A. Herrmann, Plasma Phys. Control. Fusion 44 (2002) 883.

[2] N. Klimov et al., J. Nucl. Mater. 390-391 (2009) 721.

[3] A. Loarte et al., Progress in the ITER Physics Basis Chapter 4: Power and particle control, Nuclear Fusion 47 (2007) S203.

[4] A. Loarte et al., Plasma Phys. Control. Fusion 45 (2003) 1549.

[5] P.T. Lang et al., Nuclear Fusion 44 (2004) 665.

[6] A. Kallenbach et al., J. Nucl. Mater. 337-339 (2005) 732.

[7] L.R. Baylor et al., Nuclear Fusion 47 (2007) 1598.

[8] A. Geraud et al., Fusion Engineering and Design 82 (2007) 2183.

[9] A. Geraud et al., Fusion Engineering and Design 69 (2003) 5.

[10] M.J. Watson et al., 18th IEEE/NPSS SOFE Conference, Albuquerque (1999), 326.

[11] J. Pamela et al., J. Nucl. Mater. 363-365 (2007) 1.

[12] G. Kocsis et al., Proc. 36th EPS Conference on CFPP, Sofia (2009), P1.151.

[13] A. Loarte et al., Phys. Plasmas 11 (2004) 2668. 
[14] G. Saibene et al., in Fusion Energy 2008 (Proc. 22nd Int. Conf. Geneva, 2008) (Vienna:IAEA) CD-ROM file EX/2-1 and http://www-naweb.iaea.org/napc/physics/FEC/FEC2008/html/index.htm

[15] T. Onjun et al., Physics of Plasmas 12, (2005) 012506.

[16] Y. Liang et al., Nuclear Fusion 50 (2010) 025013.

[17] T.E. Evans et al., Phys. Rev. Lett. 92 (2004) 235003-1.

[18] Y. Liang et al., Proc. 36th EPS Conference on CFPP, Sofia (2009), O5.062.

[19] P.T. Lang et al., Nuclear Fusion 43 (2003) 1110.

[20] H. Urano et al., Plasma Phys. Control. Fusion 46 (2004) A315.

[21] F.M. Poli et al., Nuclear Fusion 50 (2010) 025004.

[22] F. Poli et al., Proc. 37th EPS Conference on CFPP, Dublin (2010), O2.105.

[23] B. Alper et al., Proc. 37th EPS Conference on CFPP, Dublin (2010), P2.173.

[24] P.T. Lang et al., Nuclear Fusion 48 (2008) 095007.

[25] G.T.A. Huysmans, Proc. 37th EPS Conference on CFPP, Dublin (2010), P4.132.

[26] G.T.A. Huysmans et al., Plasma Phys. Control. Fusion 51 (2009) 124012.

[27] G. Kocsis et al., Proc. 37th EPS Conference on CFPP, Dublin (2010), P4.136.

[28] R. Wenninger et al., Proc. 37th EPS Conference on CFPP, Dublin (2010), P4.173.

[29] T. Eich et al., Plasma Phys. Control. Fusion 47 (2005) 815.

[30] F. Koechl et al., Proc. 37th EPS Conference on CFPP, Dublin (2010), O4.123.

[31] G.T.A. Huysmans et al., in Fusion Energy 2010 (Proc. 23rd Int. Conf. Daejeon, 2010)

(Vienna:IAEA) CD-ROM file THS/7-1 and

http://www-naweb.iaea.org/napc/physics/FEC/FEC2010/html/index.htm

[32] L.R. Baylor et al., Proc. 37th EPS Conference on Controlled Fusion and Plasma Physics, Dublin (2010), P2.117.

[33] N. Hayashi et al., in Fusion Energy 2010 (Proc. 23rd Int. Conf. Daejeon, 2010)

(Vienna:IAEA) CD-ROM file THS/P3-02 and

http://www-naweb.iaea.org/napc/physics/FEC/FEC2010/html/index.htm

[34] A. Loarte et al., in Fusion Energy 2010 (Proc. 23rd Int. Conf. Daejeon, 2010)

(Vienna:IAEA) CD-ROM file ITR/1-4 and

http://www-naweb.iaea.org/napc/physics/FEC/FEC2010/html/index.htm

[35] H. Thomsen et al., in Fusion Energy 2010 (Proc. 23rd Int. Conf. Daejeon, 2010)

(Vienna:IAEA) CD-ROM file EXD/6-6Rb and

http://www-naweb.iaea.org/napc/physics/FEC/FEC2010/html/index.htm

[36] R.J. Hawryluk et al., Nuclear Fusion 49 (2009) 065012.

[37] G. Kocsis et al., Nuclear Fusion 47 (2007) 1166.

[38] H.W. Müller et al., Phys. Rev. Lett. 83 (1999) 2199.

[39] B. Plöckl et al., Proc. 26th SOFT Conference, Porto (2010), P1.77.

[40] S. Maruyama et al., in Fusion Energy 2010 (Proc. 23rd Int. Conf. Daejeon, 2010)

(Vienna:IAEA) CD-ROM file ITR/P1-28 and

http://www-naweb.iaea.org/napc/physics/FEC/FEC2010/html/index.htm 